\title{
Myeloid Zinc Finger 1 (MZF1) Maintains the Mesenchymal Phenotype by Down-regulating IGF1R/p38 MAPK/ER $\alpha$ Signaling Pathway in High-level MZF1-expressing TNBC cells
}

\author{
CHIA-HERNG YUE ${ }^{1,2}$, JER-YUH LIU ${ }^{3,4}$, CHING-SHIANG CHI ${ }^{5}$, CHING-WEN HU ${ }^{6}$, \\ KOK-TONG TAN ${ }^{1}$, FU-MEI HUANG ${ }^{7}$, YING-RU PAN $^{8}$, KUN-I LIN $^{9}, 10$ and CHIA-JEN LEE ${ }^{8,11}$ \\ ${ }^{1}$ Department of Surgery, Tungs' Taichung Metroharbor Hospital, Taichung, Taiwan, R.O.C.; \\ ${ }^{2}$ Department of Nursing, Jen-Teh Junior College of Medicine, Nursing and Management, Miaoli, Taiwan, R.O.C.; \\ ${ }^{3}$ Center for Molecular Medicine, China Medical University Hospital, Taichung, Taiwan, R.O.C.; \\ ${ }^{4}$ Graduate Institute of Biomedical Sciences, China Medical University, Taichung, Taiwan, R.O.C., \\ ${ }^{5}$ Department of Pediatrics, Tungs' Taichung Metroharbor Hospital, Taichung, Taiwan, R.O.C.; \\ ${ }^{6}$ Department of Nursing, Tungs' Taichung Metroharbor Hospital, Taichung, Taiwan, R.O.C., \\ ${ }^{7}$ Operating Theatre, Chung Shan Medical University Hospital, Taichung, Taiwan, R.O.C.; \\ ${ }^{8}$ Department of Medical Research, Tungs' Taichung Metroharbor Hospital, Taichung, Taiwan, R.O.C.; \\ ${ }^{9}$ Department of Obstetrics and Gynecology, Chang Bing Show Chwan Memorial Hospital, Changhua, Taiwan, R.O.C.; \\ ${ }^{10}$ Department of Cosmetic Science, Providence University, Taichung, Taiwan, R.O.C.; \\ ${ }^{11}$ Department of Rehabilitation, Jen-Teh Junior College of Medicine, Nursing and Management, Miaoli, Taiwan, R.O.C.
}

\begin{abstract}
Background/Aim: Signaling regulation of myeloid zinc finger 1 (MZF1) has been implicated in the progression of many human malignancies; however, the mechanistic action of $M Z F 1$ in triple-negative breast cancer (TNBC) progression remains elusive. In this study, the aim was to investigate the molecular mechanisms of MZF1 and its functional role in TNBC cellular migration and invasion. Materials and Methods: Hs578T and MDA-MB-231 cells were transfected to stably express the acidic domain of MZF1 (MZF1 $60-72)$, or were transfected with MZF1-specific or ELKI-specific short hairpin RNA (shRNA). Changes in cell morphology and distributions of cellular proteins were observed and subsequently migration and invasion were measured by wound healing and transwell assays. Expression levels of epithelial-mesenchymal transition (EMT)-related genes were carried out using immunoblotting and quantitative reverse transcription-polymerase chain reaction (RT-PCR) assays. Data of transcriptional regulation
\end{abstract}

Correspondence to: Chia-Jen Lee, Department of Medical Research, Tungs' Taichung Metroharbor Hospital, Taichung, Taiwan, R.O.C. Tel: +886 930059075, e-mail: chiajenlee54@gmail.com and Kun-I Lin, Department of Obstetrics and Gynecology, Chang Bing Show Chwan Memorial Hospital, No. 6 Lugong Road, Lugang Town, Changhua County, 505 Taiwan, R.O.C. Tel: +886 47812012, e-mail: kunidg107@gmail.com

Key Words: Insulin-like growth factor 1 receptor (IGF1R), myeloid zinc finger 1 (MZF1), triple-negative breast cancer (TNBC). were obtained from promoter-luciferase reporter and chromatin immunoprecipitation (ChIP) assays. Results: Herein, we found that MZF1 in high-level MZF1-expressing TNBC cells is associated with cell migration, invasion, and mesenchymal phenotype. MZF1 interacted with the promoter region of insulinlike growth factor 1 receptor $(I G F 1 R)$ to drive invasion and metastasis of high-level MZF1-expressing TNBC cells. Exogenous expression of the acidic domain of $M Z F 1$ repressed the binding of endogenous MZF1 to IGFIR promoter via blocking the interaction with ETS-like gene 1 (ELK1). This blockage not only caused MZF1 protein degradation, but also restrained ELK1 nuclear localization in high-level MZF1expressing TNBC cells. MZF1, but not ELK1, was necessary for the retention of mesenchymal phenotype by repressing IGF1R promoter activity in TNBC cells expressing high levels of MZF1. Activation of the IGF1R-driven p38MAPK-ER $\alpha$-slug-E-cadherin signaling axis mediated the conversion of mesenchymal cell to epithelial phenotype, caused by MZF1 destabilization. These results suggest that MZF1 is an oncogenic inducer. Conclusion: Blocking of the MZF1/ELK1 interaction to reduce MZF1 protein stability by saturating the endogenous MZF1/ELK1 binding domains might be a promising therapeutic strategy for the treatment of high-level MZF1-expressing TNBC.

Breast cancer is the most common malignancy worldwide in women contributing to more than $25 \%$ of the total number of newly-diagnosed cases, and ranks second in terms of global cancer-related mortality (1). Approximately 12-17\% of breast cancer cases belong to the triple-negative subtype, 
which lacks the regular expression of estrogen receptor (ER), progesterone receptor (PR) and overexpression of human epidermal growth factor receptor type 2 (HER2, also known as ErbB2 or Neu) (2). Triple-negative breast cancer (TNBC) is classified as a poorly differentiated and aggressive type of breast cancer. Therapy for TNBC is limited to conventional chemotherapy due to the absence of a suitable target, e.g. ER, PR, and HER2. However, relapse leading to poor clinical outcome occurs constantly because of high rates of metastasis and general inaccuracy of chemotherapy $(3,4)$. This may be due to its high level of molecular heterogeneity that is another reason why TNBC cannot be effectively treated. Thus, elucidating the mechanisms of cancer progression is important for improving the therapeutic efficiency of TNBC.

The insulin-like growth factor-1 (IGF1)/IGF1 receptor (IGF1R) signaling is the major signal transduction pathway in IGF family (5). IGF1R is a transmembrane tyrosine kinase receptor that is activated through autophosphorylation after binding of IGF1, IGF2, or insulin. Signaling through the IGFR activates cascades such as RAS/MAPK and PI3K/Akt $(6,7)$. The IGF1R pathway is involved in the regulation of mitogenesis, apoptosis, development, epithelial-tomesenchymal transition (EMT)-associated tumor metastasis, hematological malignancies, and drug resistance $(8,9)$. In some subtypes, IGF1R levels correlate with a favorable prognosis, while in others it is associated with recurrence and poor prognosis, suggesting different actions based upon cellular and molecular contexts (6). However, understanding of the important components of the IGF1R signaling pathway and the instances where crosstalk and compensation can occur in breast cancer, remain to be explored. Human IGF1R gene is located on chromosome 15 (15q26.3), and multiple tumor suppressor genes such as p53 and BRCA1 repress the IGFIR expression at the transcription level in TNBC (8). In fact, the transcriptional regulators and underlying mechanisms of the implication of IGF1R in TNBC are limitedly identified.

In this study, using the TRANSFAC database of eukaryotic transcription factors and chromatin immunoprecipitation (ChIP), we identified the putative MZF1 binding sequences within the IGF1R promoter. MZF1 has been found as a core transcription factor that was involved in the regulation of gene expression, as well as in the metastasis of breast cancer (10). We also demonstrated that TNBC cell lines expressing high levels of MZF1 mRNA exhibit a high expression of MZF1 at the protein level. In an earlier study, we have shown that overexpression of the peptide fragment including the acidic domain of MZF1 (MZF1 $\left.{ }_{60-72}\right)$ and the heparin-binding domain of ELK1 (ELK1 ${ }_{145-157}$ ) decreases DNA-binding ability of endogenous MZF1 to the PKC $\alpha$ promoter and rearranges its transcription (11). In support of these findings, phosphorylation of MZF1 has been shown to mediate EMT by elevating the expression of $\mathrm{N}$-cadherin in human esophageal cancer cells
(12). Collectively, earlier observations have suggested that MZF1 might be crucial for the regulation of metastasis in malignant tumor cells. Here, we found that blocking the binding of MZF1 with ELK1 caused MZF1 protein degradation and restrained ELK1 nuclear localization in highlevel MZF1-expressing TNBC cells. Recently, EMT and mesenchymal-epithelial transition (MET) have been suggested as possible mechanisms involved in breast cancer metastasis (13). Thus, in this study, we further investigated whether MZF1 is necessary for maintaining the partial mesenchymal phenotype by repressing IGF1R promoter activity in high-level MZF1-expressing TNBC cells. Degraded MZF1 lost its ability to bind directly to IGF1R promoter regulatory sequences, and consequently, IGF1R transcription was up-regulated. The activation of IGF1R-driven p38 mitogen-activated protein kinase (p38 MAPK)-ER $\alpha$-slug-E-cadherin signaling axis mediated the conversion of mesenchymal cell to epithelial phenotype caused by destabilization of MZF1. Taken together, our findings indicated that in MZF1 is implicated in the modulation of TNBC cell motility and invasion potential, and functions through the suppression of IGF1R expression by direct binding to its promoter region.

\section{Materials and Methods}

Microarray analysis. The data mentioned in this study have been deposited in the National Center for Biotechnology Information (NCBI) Gene Expression Omnibus and can be accessed through Gene Expression Omnibus Series accession number GSE56306 (http://www.ncbi.nlm.nih.gov/geo).

Immunohistochemical (IHC) staining. Tissue array slides including 208 cases of breast cancer (\#BR20834), 205 invasive ductal carcinomas, 2 invasive lobular carcinomas, and 1 invasive papillary carcinoma; 15 invasive ductal carcinomas were lost during the evaluation) and 30 cases of TNBC (\#BR1503b), 7 intraductal and 60 invasive ductal carcinomas, which contained 30 TNBC, duplicate cores per case) were purchased from US Biomax Inc. (Rockville, MD, USA). Detailed information for this array is available at http://www.biomax.us/tissue-arrays/. The sections were immunostained against MZF1 (1:400, ab64866, Abcam) as previously described (14). The representative staining results for samples were scored by visual assessment as "weak," "moderate," or "strong" according to staining intensity, as previously described (11). Moderate or strong expression of MZF1 protein was given a positive rating, otherwise rating was negative.

Cell lines. Human breast cancer cell lines were obtained from the Bioresources Collection and Research Center, Food Industry Research and Development Institute, Hsinchu, Taiwan. Specifically, three TNBC (ER-/PR-/HER2-) cell lines, namely Hs578T, MDAMB-231 and MDA-MB-468, as well as the luminal-type $\left(\mathrm{ER}^{+} / \mathrm{PR}^{+} / \mathrm{HER}^{-}{ }^{-}\right) \mathrm{MCF}-7$ cell line and the HER2-enriched

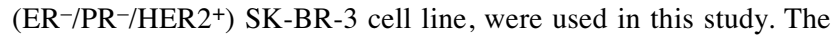
MDA-MB-231, MDA-MB-468, MCF-7, SK-BR-3 cells were cultured in DMEM/F-12 medium (HyClone, Logan, UT, USA), and the Hs 578T cells were cultured in DMEM medium (HyClone). 
Cells were maintained in medium specific for each cell line with containing $10 \%$ fetal bovine serum, 100 units $/ \mathrm{ml}$ penicillin, and 100 $\mu \mathrm{g} / \mathrm{ml}$ streptomycin (Invitrogen) in a humidified atmosphere containing $5 \% \mathrm{CO}_{2}$ at $37^{\circ} \mathrm{C}$.

Immunoblotting and co-immunoprecipitation (co-IP) analysis. For western blotting, cells were lysed in RIPA buffer (50 mM Tris- $\mathrm{HCl}$ pH8.0, $150 \mathrm{mM} \mathrm{NaCl}, 0.1 \%$ SDS, $0.5 \%$ sodiumdeoxycholate, $1 \%$ TritonX-100, $10 \mathrm{mM} \mathrm{MgCl}$, and $5 \mathrm{mM}$ EDTA) containing protease and phosphatase inhibitors (15). Anti-MZF1 (sc-293218), anti-ELK1 (sc-365876), anti-Ki-67 (sc-23900), and anti- $\beta$-actin (sc-69879) antibodies were purchased from Santa Cruz Biotechnology (Santa Cruz, CA, USA). Anti-phospho-ELK1 (\#9181), anti-Lamin B1 (\#12586), anti-IGF1R (\#3027), anti-ER $\alpha(\# 8644)$, anti-E-cadherin (\#3195), anti-Vimentin (\#5741), anti-Slug (\#9585), anti-p38 MAPK (\#8690), and anti-phospho-p38 MAPK (\#9215) antibodies were from Cell Signaling (Beverly, MA, USA). Anti-mouse and antirabbit HRP-conjugated secondary antibodies were purchased from Promega. The inhibitors SD203580 and AG-1024 were obtained from Cell Signaling and Cayman (Ann Arbor, MI, USA). The protein levels were quantified by using Image J software (NIH, Bethesda, MD, USA) and protein level was indicated as target protein level/ $\beta$-actin level. For co-IP, cells were lysed with cold lysis buffer $\left(142.5 \mathrm{mM} \mathrm{KCl}, 5 \mathrm{mM} \mathrm{MgCl}{ }_{2}, 10 \mathrm{mM}\right.$ HEPES $\mathrm{pH} 7.2$, $0.2 \%$ Nonidet P-40, and $1 \mathrm{mM}$ EGTA) containing $2 \mathrm{mM}$ phenylmethylsulfonyl fluoride (PMSF), $2 \mu \mathrm{g} / \mathrm{ml}$ aprotinin, $2 \mu \mathrm{g} / \mathrm{ml}$ leupeptin, $1 \mathrm{mM} \mathrm{NaF}$, and $1 \mathrm{mM} \mathrm{Na} \mathrm{VO}_{4}$. Lysates were then centrifuged at $16,000 \mathrm{~g}$ for $10 \mathrm{~min}$ at $4^{\circ} \mathrm{C}$. Equal amounts of protein extracts were further analyzed by co-IP and immunoblotting following methods described previously (16). Approximately 500 $\mu \mathrm{g}$ of each lysate were subjected to co-IP with the appropriate specific antibodies. Anti-MZF1 (ab64866) antibody was from Abcam (Cambridge, UK). After incubating overnight at $4^{\circ} \mathrm{C}$, the specific proteins were precipitated with proteinA/G sepharose beads (Thermo Fisher Scientific, Rockford, IL, USA) and purified using according to the manufacturer's protocol. Beads were eluted in $2 x$ SDS-PAGE sample buffer and immunoblot analysis was performed. NE-PER nuclear and cytoplasmic extraction reagents (Thermo Fisher Scientific) were used for separation and preparation of cytoplasmic and nuclear extracts from breast cancer cells.

Plasmid construction. The pcDNA 3.1/myc-His expression vector, as vector control, was obtained from Invitrogen (Carlsbad, CA, USA). Open reading frame of the human MZF1 gene (GenBank Accession No.AF161886, 10781-12235 bp) was amplified from the Hs578T cells by reverse transcirption-polymerase chain reaction (RT-PCR); then, it was cloned into the pcDNA 3.1/myc-His vector (Invitrogen), as previously described (17), while empty vectors were used as vehicle control. The resulting recombinant plasmids carrying the $M Z F 1$ gene or the $M Z F 1_{60-72}$ gene fragment were designated pcDNA-MZF1 and pcDNA-MZF1 $1_{60-72}$, respectively. Small hairpin RNA specific for $E L K 1$ (shELK1; cloneID: TRCN0000007450-TRCN0000007454) and shRNA specific for MZF1 (shMZF1; cloneID: TRCN0000017133TRCN0000017137) were cloned into pLKO.1 vector plasmids (National RNAi Core Facility at Academic Sinica, Taipei, Taiwan). Empty pLKO.1 vectors were used as control for shRNA.

Transfection and stable clone establishment. The various doses of plasmids, as indicated, were transfected into Hs578T or MDAMB-231 cells using Lipofectamine 2000 transfection reagent
(Invitrogen). After incubating for $48 \mathrm{~h}$, the cells were harvested and assayed for subsequent experiments. Stable clones were established by seeding low-passage cells and transfected with the plasmid encoding shELK1, shMZF1 or MZF1 $1_{60-72}$ using Lipofectamine 2000. Forty-eight hours post-transfection, the cells were maintained in medium supplemented with geneticin (G418 sulfate; AMRESCO, Solon, OH, USA) or puromycin (AMRESCO) at $37^{\circ} \mathrm{C}$ for 4 weeks. Individual clones were then transferred to $24-$ well plates and grown until confluence. Two different cell clones stably expressing MZF160-72 were selected for further analysis. After being transferred to $10-\mathrm{cm}$ dishes, the cells were cultured until confluence, harvested, and frozen in liquid nitrogen for further experiments.

Cell proliferation, migration, and invasion assays. Viability of cells expressing MZF1 $1_{60-72}$, shMZF1, shELK1, or control vector was detected by 3-[4,5-dimethylthiazol-2-yl]-2,5-diphenyltetrazoliumbromide (MTT) assay as described previously (16). Briefly, cells were seeded in 24-well plates at $1 \times 10^{4}$ cells/well and cultured in DMEM containing $10 \% \mathrm{FBS}$ at $37^{\circ} \mathrm{C}$. Cell proliferation was determined at 16 and $24 \mathrm{~h}$ after seeding by adding MTT and incubating the cells further for $3 \mathrm{~h}$. Migration assay was performed using a 48-well Boyden chamber (NeuroProbe, Gaithersburg, MD, USA) plated with $8 \mu \mathrm{m}$ pore size polycarbonate membrane filters (NeuroProbe). The lower compartment was filled with DMEM containing $20 \%$ FBS. Cells were placed in the upper part of the Boyden chamber and incubated for $16 \mathrm{~h}$. After incubation, the cells were fixed with methanol and stained with $0.05 \%$ Giemsa for $1 \mathrm{~h}$. The cells on the upper surface of the filter were removed with a cotton swab. The filters were then rinsed in distilled water until no additional stain leaching was observed. The cells were then airdried for $15 \mathrm{~min}$. The migratory phenotypes were determined by counting the cells that migrated to the lower side of the filter through microscopy at $200 \times$ magnification. For each membrane, a total of 4 random fields were selected and counted, and each sample was assayed in triplicate. The invasion assay was performed using a 48-well Boyden chamber with polycarbonate filters. The upper side was pre-coated with $10 \mu \mathrm{g} / \mathrm{ml}$ Matrigel (CollaborativeBiomedical, Bedford, MA, USA). Cells were placed in the upper part of the Boyden chamber and incubated at $37^{\circ} \mathrm{C}$ for $24 \mathrm{~h}$. The experimental procedures were identical to the migration assay procedures.

Immunofluorescence microscopy. Hs578T cells were plated on glass coverslips and incubated in complete medium over night at $37^{\circ} \mathrm{C}$. The cells were washed twice with ice-cold PBS, fixed in $3.7 \%$ paraformaldehyde for $20 \mathrm{~min}$, and then permeabilized with $0.1 \%$ saponin, $2 \%$ goat serum (VectorLaboratories, Peterborough, UK) and $0.02 \% \mathrm{NaN} 3$ at RT for 20 min. ELK1 was visualized using an antiELK1 antibody (1:400) (SantaCruz) followed by a FITC-conjugated goat anti-rabbit IgG antibody (RocklandImmunochemicals, Gilbertsville, PA, USA). MZF1 was visualized using an anti-MZF1 antibody (clone1F7, 1:400) (SantaCruz) followed by a rhodamineconjugated goat anti-mouse IgG antibody (Rockland Immunochemicals). Cells on coverslips were mounted by using the ProLong Antifade kit (MolecularProbes, Eugene, Oregon). Immunofluorescence density and images were obtained using a Leica TCS SP2 confocal microscope detection system (Wetzlar, Germany) at excitation wave lengths of 488 and $543 \mathrm{~nm}$. Emissions were detected using bandpass filters of $505 \mathrm{~nm}$ to $525 \mathrm{~nm}$ and $578 \mathrm{~nm}$ to $623 \mathrm{~nm}$. 
Protein stability assay. The stability of MZF1 and ELK1 in Hs578T and MDA-MB-231 cells stably expressing MZF1 ${ }_{60-72}$ was determined using a translational inhibitor. Analysis of MZF1 and ELK1 proteins were performed in the presence of cycloheximide as previously described (18). Briefly, cells stably expressing MZF $1_{60}$ 72 or control vector were treated with $50 \mu \mathrm{g} / \mathrm{ml}$ cycloheximide (Sigma-Aldrich, StLouis, MO, USA) for 2, 4 and up to $8 \mathrm{~h}$. Cells were lysed in RIPA buffer containing protease and phosphatase inhibitors. The expression of MZF1, ELK1 and $\beta$-actin (loading control) analyzed by Western blotting as described above.

$R N A$ isolation and reverse transcriptase-PCR. Using TRIOL reagent (Invitrogen), total cellular RNA was isolated from Hs578T or MDAMB-231 cells stably expressing shMZF1 or control vector, and transiently transfected with MZF1 plasmid or vector only. RT-PCR was performed as described previously (15). The following primers were used: MZF1 forward, 5'-AGGTCCAGGTAGTGTAAGCCCT3'; MZF1reverse, 5'-ACTCTCCGATGCTCTTCCAG-3' (yielding a 495-bp PCR product) (19); IGF1R forward, 5'-CCATTCTCATGCC TTGGTCT-3'; IGF1Rreverse, 5'-TGCAAGTTCTGGTTGTCGAG3' (yielding a 113-bp PCR product) (20); $\beta$-actin forward, 5'TTCCAGCCTTCCTTCCT-3'; $\beta$-actin reverse, 5 '-GAAGGCTTATT CCAGTTT-3' (yielding a 457-bp PCR product) (20). The PCR products were analyzed by $1.5 \%$ agarose gels and visualized by ethidium bromide staining.

Luciferase reporter assay. To construct the reporter plasmid, IGF1RLuc containing the human IGF1R gene promoter was amplified by PCR with the template from genomic DNA of Hs578T cells. The primers used for PCR reaction were as follows: IGF1Rf (forward, 5'cggggtaccCTCTCCTCGAGCCACTCTGGGC-3') and IGF1Rr (reverse,5' '-ggaagatctGCCCCGAAGTCCGGGTCACA-3'). The PCR product containing IGF1R gene promoter $(-765 /+583)$ was digested using KpnI and BglII restriction enzymes (New England Biolabs, Ipswich, MA, USA), the DNA fragment was ligated into the pGL3basic vector (Promega, Madison, WI, USA) and then the positive clone, IGF1R-Luc, was obtained via selection. The reporter gene assay of IGF1R promoter was performed using Hs578T and MDA-MB-231 cells. The appropriate number of cells was transfected with sufficient reporter plasmid, IGF1R-Luc or pGL3 control vector plasmid. To normalize the transfection efficiency, the pCH110 plasmid (Amersham, Piscataway, NJ, USA) carrying the $\beta$-galactosidase gene was also co-transfected. Hs578T and MDA-MB-231 cells stably expressing MZF1 $_{60-72}$, shELK1, shMZF1 or control vector were transiently co-transfected with pGL3-IGF1R-Luc or pGL3 control vector and pCH110 plasmids. Transfected cells were harvested $48 \mathrm{~h}$ after transfection, and the luciferase and $\beta$-galactosidase activity assays were performed. The luciferase activity in the cell lysates was determined using a Dual Luciferase Assay System (Promega, Madison, WI, USA) according to the manufacturer's instructions. The measured values of luciferase activity were normalized to that of $\beta$-galactosidase activity to generate relative luciferase activity.

Chromatin immunoprecipitation (ChIP) assay. ChIP was performed using the Agarose ChIP Kit (Thermo Fisher Scientific), according to the manufacturer's instructions. Briefly, Hs578T cells were lysed, and DNA was extracted, precipitated with with an antibody specific for MZF1 and dissolved in $50 \mu$ of Tris buffer (TB, $10 \mathrm{mM}$ Tris- $\mathrm{HCl} \mathrm{pH} 8.0$ ). Immunoprecipitated DNA was analyzed by PCR. The region of the IGF1R promoter was amplified from the immuno-precipitated chromatin using the primers: forward (a), 5'-GCGGGGGCATTGTTTTTGGA-3', and reverse (a), 5'-CCCGGT TCCCCAAGACGTG-3' (product size: 203-bp); forward (b), 5'-GCGCGTGTCTCTGTGTGC-3', and reverse (b), 5'-CGGAGTT AATGCTGGTAAACAA-3' (product size: 303-bp); forward (c), 5'-GTGTGTGTCCTGGATTTGGGA-3', and reverse (c), 5'-GCCC CGAAGTCCGGGTCACA-3' (product size: 168-bp). The PCR products were analyzed by $1.5 \%$ agarose gels and visualized by ethidium bromide staining. For re-ChIP, a previously described methodology was followed (21). The precipitated complexes eluted from the primary ChIP (from four reactions) were pooled and incubated with $50 \mu \mathrm{l}$ ChIP elution buffer (50 mM NaHCO $3,1 \%$ SDS). The samples were mixed for $30 \mathrm{~min}$ at room temperature and centrifuged, and the supernatants were collected. The complexes were eluted twice, and both eluates were combined. The pooled eluates were diluted 1:10 in a buffer (1\% TritonX-100, $5 \mathrm{mM}$ EDTA, $150 \mathrm{mM} \mathrm{NaCl}$, and $25 \mathrm{mM}$ Tris- $\mathrm{HCl}$, pH8.0) containing a protease inhibitor mixture (Roche Diagnostics, Mannheim, Germany). Further supernatant re-ChIP assays and result analysis were performed with with an antibody specific for ELK1 as previously described for primary ChIP immunoprecipitation.

Statistical analysis. The data are expressed as mean \pm standard deviation (SD) of three independent experiments and were analyzed by ANOVA using the GraphPad prism software program (Graph Pad Software, La Jolla, CA, USA). The Kaplan-Meier curves were used to assess the effect of single gene on breast cancer prognosis. Pearson's chi-square test and Student's $t$-test were used in two-group comparisons. All $p$ values $<0.05$ was considered as statistically significant.

\section{Results}

Elevated MZF 1 expression was correlated with the mortality of $T N B C$. In order to evaluate whether MZF1 status is associated with breast cancer prognosis, the mRNA expression data of breast cancer patients were downloaded from the Kaplan-Meier plotter microarray database (http://kmplot.com/ analysis/index. php?p=service \&cancer=breast) and were analyzed (univariate Kaplan-Meier method) (22). In total, 274 breast cancer patients, including 198 patients with TNBC and 76 patients with triplepositive breast cancer (TPBC; ER/PR/HER2 positive) were analyzed. Overall survival (OS) curves are depicted in Figure 1A. In the TPBC group, patients with high MZF1 levels tended to exhibit better clinical outcomes than those with low MZF1 levels (Figure 1A, Log rank test $p=0.023$ ). However, in the TNBC group, patients with high MZF1 levels demonstrated poorer OS than those with low MZF1 expression (Figure 1A, Log rank test $p=0.038$ ). Unlike TPBC, we found that high tumor expression of MZF1 was associated with reduced OS in TNBC $(p<0.05)$. On this basis, it is reasonable to assume that MZF1 plays a different role in different subtypes of breast cancer.

Moreover, to compare the clinical relevance between MZF1 expression and TNBC or BC patients, the expression levels of MZF1 protein were examined in tissue arrays by IHC. The moderate-to-strong staining of MZF1 was observed in 22/30 $(73.3 \%)$ and $151 / 190(79.5 \%)$ of TNBC and BC patients, respectively. Thus, no difference was observed in the percentages of "moderate-to-strong" MZF1 expression between TNBC and other subtypes of breast cancer. 

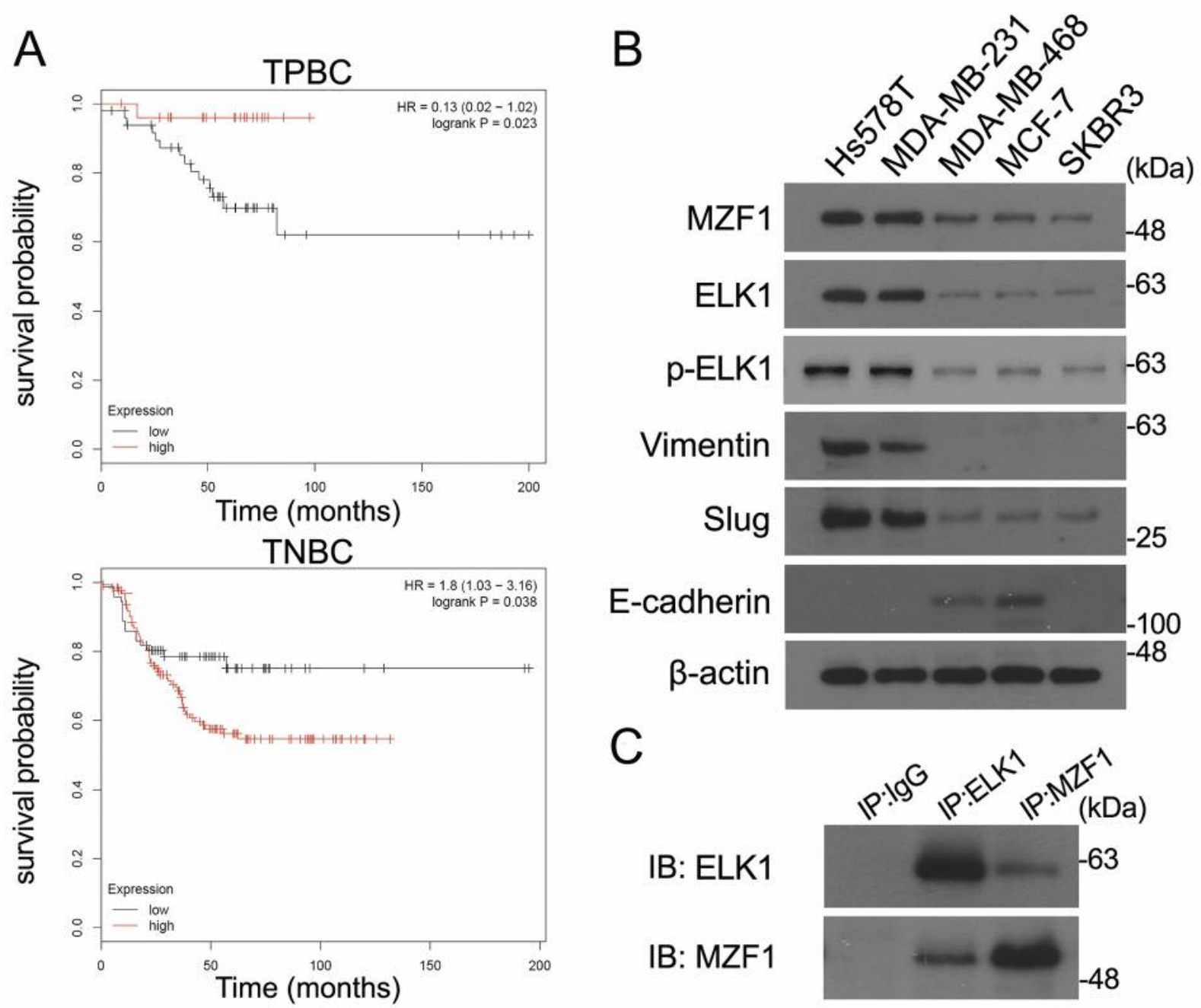

Figure 1. MZF1 overexpression is associated with poor prognosis in patients with triple-negative breast cancer (TNBC). Survival curve for different MZF1 expression levels in patients with triple-positive breast cancer (TPBC) were analyzed using Kaplan-Meier plotter microarray database (upper panel). Kaplan-Meier curve analysis showing overall survival of patients with TNBC stratified by MZF1 expression level (lower panel) (A). Immunoblotting analyses of MZF1 and EMT markers expression in 5 human breast cancer cell lines (B). Co-IP assay of the interaction between endogenous MZF1 and ELK1 in Hs578T cells. Protein extracts were immunoprecipitated (IP) with anti-MZF1, anti-ELK1antibody, or control IgG as indicated. The resulting immunoprecipitates were resolved via SDS-PAGE and immunoblotted (IB) with both antibodies sequentially $(C)$.

Elevated MZF1 expression may be related to the EMT potential of high-level MZF1-expressing TNBC cells. The protein expression levels of EMT markers, MZF1, and ELK1 were examined in cultured TNBC, luminal-type, and HER2-enriched cell lines. Two MZF1 isoforms of molecular masses of 54 and $82 \mathrm{kDa}$ have been described to date (23). However, only the 54 $\mathrm{kDa}$ isoform of MZF1 was detected by immunoblot in the Hs578T cellular extract (data not shown). As shown in Figure 1B, MZF1 protein levels were significantly higher in the Hs578T (primary carcinosarcoma of the breast) and MDA-MB231 (invasive ductal carcinoma with metastasis) TNBC cell lines, compared to the HER2-enriched SKBR3 cells (3.96-fold; $p<0.01$ and 4.31-fold; $p<0.01$, respectively) and the ER/PR- postive MCF7 cells (2.18-fold; $p<0.01$ and 2.32-fold; $p<0.01$, respectively). Similarly, ELK1, as well as the mesenchymal markers, Vimentin and Slug, had increased expression in TNBC cell lines, compared to the non-TNBC (SKBR3 and MCF7cells). On the contrary, the epithelial marker E-cadherin was not detected in the two TNBC cell lines, Hs578T and MDA-MB-231, while it was expressed by the non-TNBC cells.

To evaluate whether MZF1 could interact with ELK1, as well as their physical interaction, co-IP assay was conducted using Hs578T and MDA-MB-231 cell lysates. The interaction between the two proteins was verified by co-IP of MZF1 by anti-ELK1 antibody and vice versa. The results indicated that MZF1 was bound to ELK1; thereby forming a protein complex 
in Hs578T (Figure 1C) and MDA-MB-231 cells (data not shown). Taken together, these data supported that MZF1/ELK1 may be implicated in the regulation of the EMT potential of the studied TNBC cells expressing high levels of MZF1.

Interruption of binding of MZF1 to ELK1 suppressed the migration and invasion in TNBC cells. Hs578T cells were stably transfected to express the acidic domain $M Z F 1_{60-72}$, in order to block the binding of endogenous MZF1 to ELK1. It was shown that the morohology of the tranfected cells (hereafter referred to as Hs578T-MZF1 $60-72$ ) was altered (Figure 2A). Conversely, vector control and parental cells resembled fibroblast-like morphology. In addition, expression level of MZF1 protein decreased simultaneously in the Hs578T$\mathrm{MZF1}_{60-72}$ cells (Figure 2A). The distinct morphology of Hs578T-MZF1 ${ }_{60-72}$ cells was reminiscent of cells that have been displayed epithelial characteristics. To investigate possible mechanisms crucial for reversing changes of mesenchymal phenotype, we examined the effects of $M Z F 1_{60-72}$ on the motility and invasion potential of TNBC cells. A significant decrease of migration through the polycarbonate membrane was observed in Hs578T-MZF1 ${ }_{60-72}$ cells, compared to the control cells ( $87.3 \%$ reduction, $p<0.01$; Figure $2 \mathrm{~B}$ ). A similar decrease was also observed for Hs578T-MZF1 ${ }_{60-72}$ invasion through the Matrigel-coated membrane $(89.1 \%$ reduction, $p<0.01$; Figure 2B). MTT viability assay showed that $M Z F 1_{60-72}$ did not affect cell viability within 16 and $24 \mathrm{~h}$ after plating.

Moreover, the expression of the proliferation marker Ki67 was evaluated in Hs578T-MZF1 $60-72$ cells. High Ki-67 expression has been associated with poor prognosis in breast cancer (24). Moreover, the expression levels of Ki-67 have been shown to be relatively higher in TNBC than in nonTNBC sections (25); therefore, we used Ki-67 to evaluate the biological response of TNBC cell lines expressing different levels of MZF1 protein However, no changes were observed in cell proliferation (Figure 2B)

Interruption of MZF1 binding to ELK1 changed their cellular localization. Confocal laser scanning microscopy (CLSM) analysis was used to invetsigate the intracellular distribution as well as the differential expression of MZF1 in Hs578T, Hs578T-MZF1 $60-72$, and vector control (Hs578T-v) cells. In the Hs578T-MZF1 ${ }_{60-72}$ cells, both accumulation of cytoplasmic ELK1 and reduced ELK1 nuclear localization were observed, compared to vector control or parental cells. In addition, CLSM analysis showed very low, or almost undetectable levels of MZF1 expression in Hs578T-MZF1 $60-72$ cells (Figure 2C). The subcellular localization of ELK1 was also verified via immunoblot analysis of its expression in nuclear protein fractions (Figure 2D). It has been reported that phosphorylation of ELK1 promotes its nuclear translocation, indicating that the inhibition of ELK1 phosphorylation may prohibit this translocation (26). In line with this evidence, our results of immunoblotting (Figure 2D) revealed very low or almost undetectable levels of phospho-ELK1 in the nuclear fraction of Hs578T-MZF1 $60-72$ cells compared to vector control or parental cells.

Interruption of MZF1 binding to ELK1 modulated the expression of EMT markers. Since $\mathrm{MZF}_{60-72}$ overexpression inhibited migration and invasion of Hs578T cells, we aimed to examine whether it also affected EMT in Hs578T-MZF1 $60-72$ cells. Therefore, the expression of mesenchymal and epithelial markers was investigated. The experiment was repeated at least 3 times and the results showed that Hs578T-MZF1 ${ }_{60-72}$ cells had reduced protein levels of mesenchymal markers (Slug and Vimentin, by $86.4 \%, p<0.05$ and $80.2 \%, p<0.05$, respectively), and increased expression of the epithelial marker E-cadherin (by 9.39-fold, $p<0.01$ ) (27) compared to the vector control cells (Figure 2D). In general, alterations in the expression of EMT

Figure 2. Disrupting the interaction between $M Z F 1$ and ELK1 by $M_{Z F 1_{60}}$ 72 interrupts EMT in Hs578T cells. Phase contrast microscopy images revealed changes in morphology from spindle-shaped to cobblestone-like

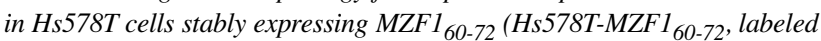
as Hs578T-M and Hs578T-M', two different stable cell clones expressing

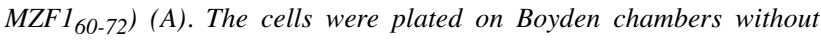
Matrigel for migration assay or with Matrigel for invasion assay and then collected after $16 \mathrm{~h}$ for migration assay (left panel) or $24 \mathrm{~h}$ for invasion assay (right panel). Migrating cells were imaged under a phase contrast microscope (upper panel). Hs578T cells were used as the control. Each bar represents the mean $\pm S D$ calculated from three independent experiments. ${ }^{*} p<0.01$ compared to the control. The effect of $M Z F 1_{60-72}$ on cell proliferation after 16 and $24 \mathrm{~h}$ was determined via an MTT assay (bottom panel). Absorbance values obtained from $H$ s578T cells were taken as $100 \%$. The effect of $M Z F 1_{60-72}$ on cell proliferation after 16 and $24 \mathrm{~h}$ was determined via immunoblotting with Ki-67 antibody (bottom panel) (B). Immunofluorescence staining showed the distribution of the ELK1 and $M Z F 1$ proteins. The cells were fixed and stained with antibodies against ELK1 and MZF1 followed by the appropriate FITC-or rhodamineconjugated secondary antibodies. Confocal slices of 0.5 and $0.6 \mu \mathrm{m}$ were obtained, and images were taken through the center of the nucleus. " $N$ " indicates the nucleus, and " $C$ " indicates the cytosol $(C)$. Western blot results showed decreased MZF1 and ELK1 expression in Hs578T-M and Hs578T-M' cells compared to Hs578T-v cells, which contained pcDNA3.1 vector control. The effect of $M Z F 1_{60-72}$ expression on the nuclear translocation of MZF1 and ELK1 was also examined. “ $(N)$ " indicates the nuclear fraction extracts, while the others are from whole-cell lysates. EMT-associated markers were also detected via western blot, and the results showed decreased Slug and Vimentin, but increased E-cadherin in Hs578T-M cells, compared to the control Hs578T-v cells. $\beta$-actin levels were used as loading controls $(D)$. Cycloheximide pulse-chase analysis of MZF1 and ELK1 stability was performed in Hs578T-v and Hs578T-M cells (upper panel). The cells were incubated with the translational inhibitor cycloheximide for the indicated time periods, and the levels of MZF1 and ELK1 were analyzed via western blot. MZF1/ELK1 cycloheximide pulsechase analysis in Hs578T-v and Hs578T-M cells in the presence or absence of the proteasome inhibitor MG132 with analysis of western blot (bottom panel). Experiments were performed in triplicate with similar results $(E)$. 
A

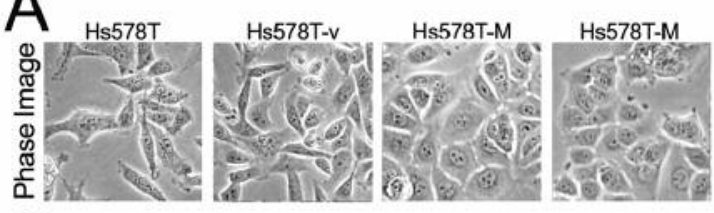

C

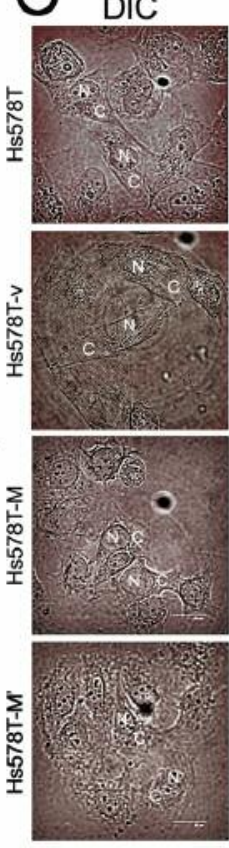

D
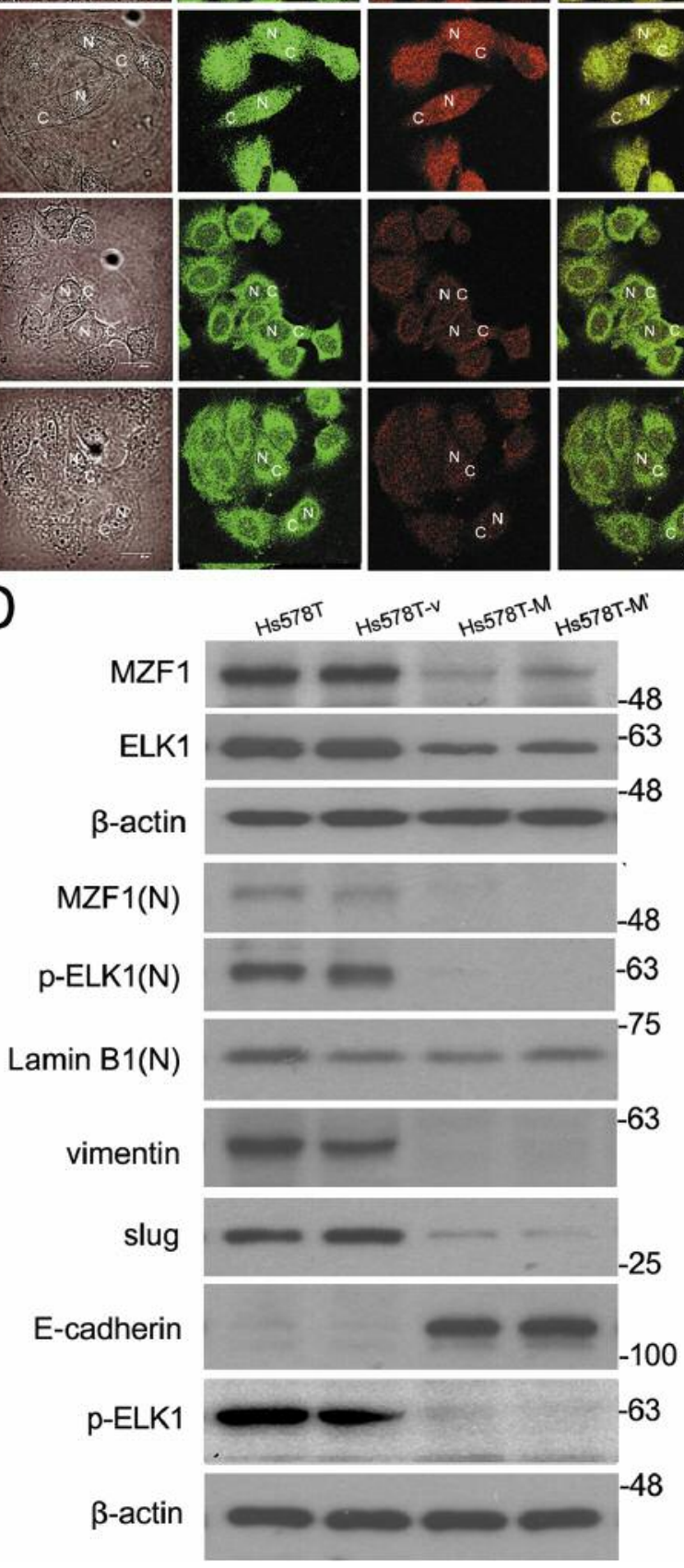

(6) No
B
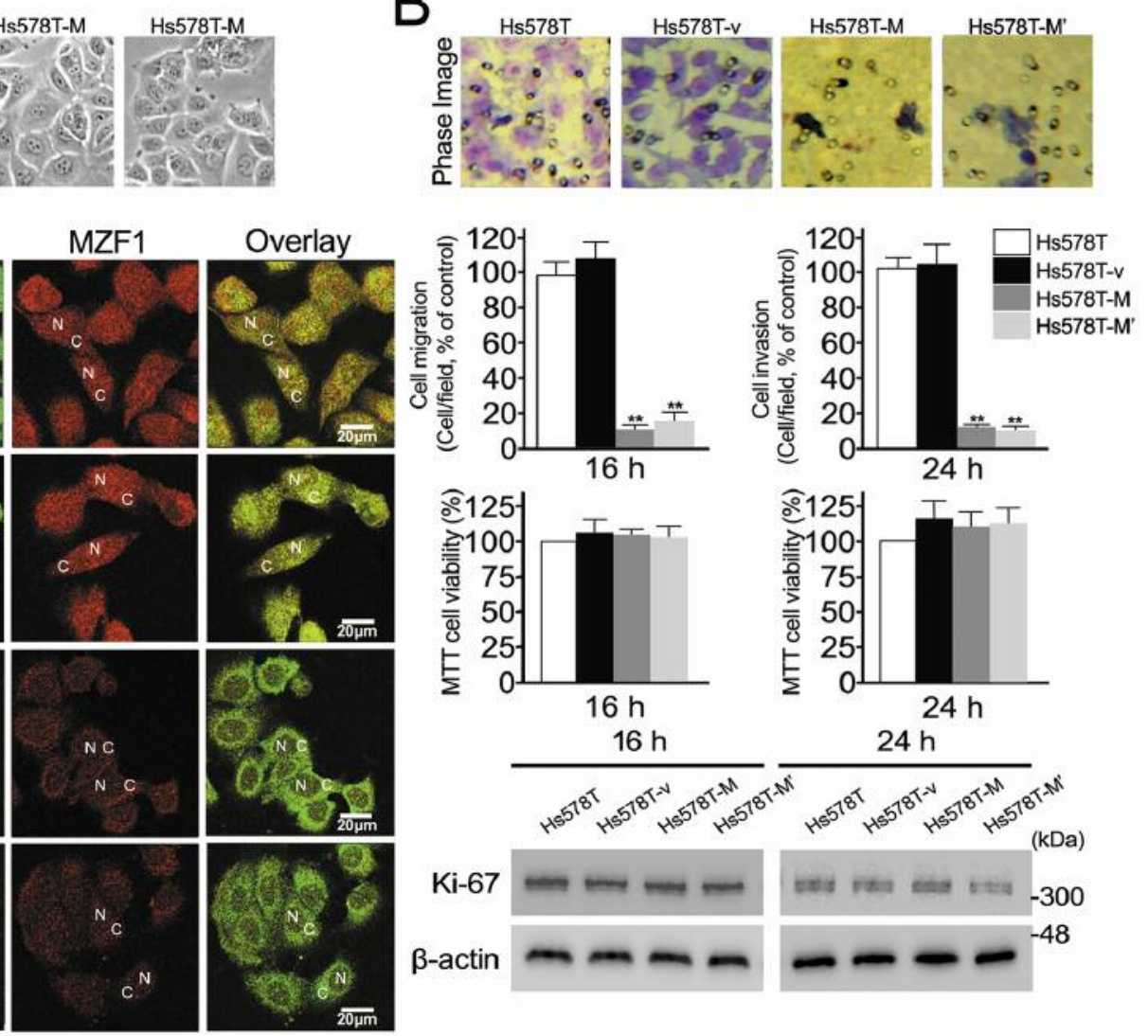

$16 \mathrm{~h}$

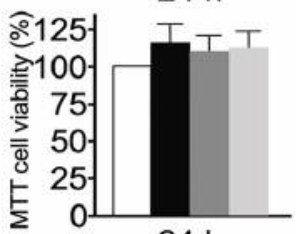

$24 \mathrm{~h}$

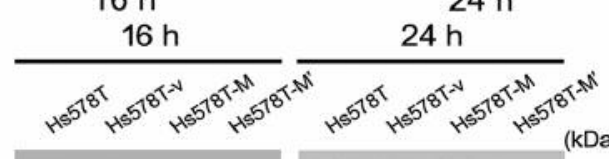

Ki-67

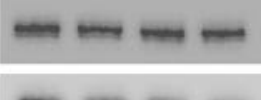

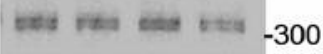

$\beta$-actin $\rightarrow-\infty$

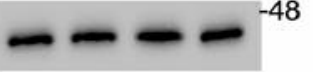

E

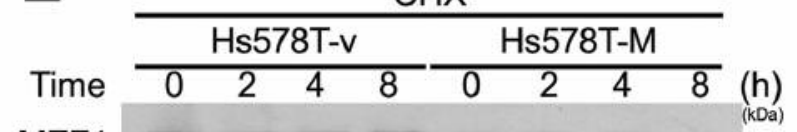

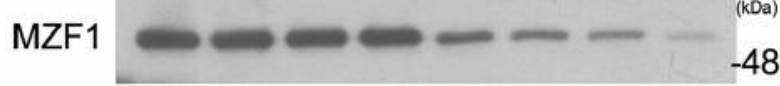
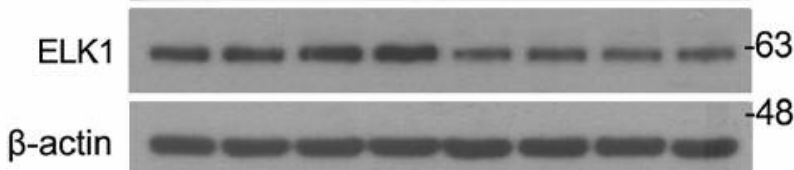

$-48$

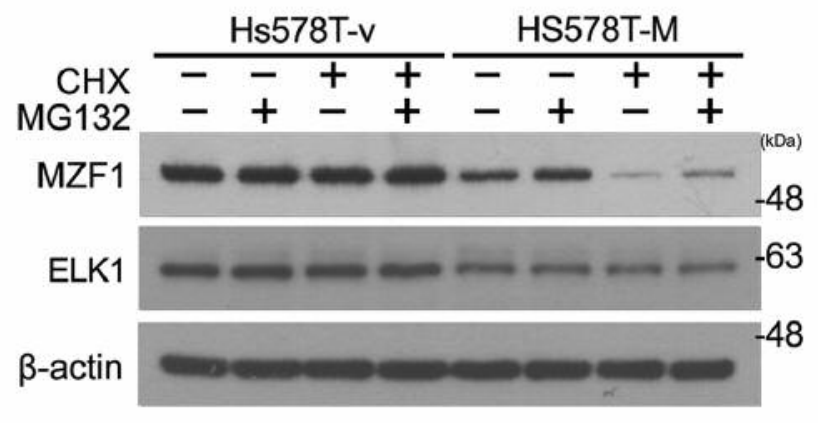


markers were partly coincident with the observed EMT-related phenotypic changes in Hs578T-MZF1 $60-72$ cells, providing additional evidence to support $\mathrm{MZF} 1_{60-72}$-mediated EMT reversal in Hs578T-MZF1 $60-72$ cells.

Blocking MZF1 and ELK1 protein complex formation modulated MZF1 proteins stability in Hs578T cells. As already shown above, expression levels of proteins MZF1 and ELK1 were decreased in Hs578T-MZF1 $60-72$ cells (Figure 2D). These observations also suggested differences in the protein stability of endogenous MZF1 in Hs578T-MZF1 ${ }_{60-72}$ cells. To test this hypothesis, the stability of the MZF1 and ELK1 proteins were analyzed via cycloheximide pulse-chase assays. As shown in Figure 2E, the MZF1 protein levels in Hs578T-MZF1 ${ }_{60-72}$ cells declined quickly after treatment with cycloheximide $(79.17 \%$ reduction compared to vector control cells, $p<0.01$, after $8 \mathrm{~h}$ of cycloheximide treatment), and displayed much shorter half-life than ELK1 (47.5\% reduction compared to vector control cells, $p<0.05$, after $8 \mathrm{~h}$ treatment with cycloheximide), which might be involved in the control of downstream gene regulation. To determine whether MZF1 degradation is mediated by the proteasome pathway, Hs578T-MZF1 ${ }_{60-72}$ cells were treated with the proteasome inhibitor MG132 for $4 \mathrm{~h}$, and then the levels of MZF1 were analyzed. A significant increase (2.11-fold; $p<0.05$ ) in MZF1 levels was observed (Figure 2E). Our data confirmed that MZF1 degradation was mediated through the proteasomedependent pathway and loss of this interaction leads to rapid degradation of MZF1 protein in $\mathrm{MZF}_{60}$-72-overexpressing Hs578T cells. These results imply that $\mathrm{MZF1}_{60-72}$ has a functional role, which is associated with regulation of protein stability and degradation. Therefore, the interaction between the endogenous MZF1 and ELK1 protein promoted the stability of MZF1 and their entrance into the cellular nucleus.

MZF1 but not ELK1 specifically regulated EMT in Hs578T and MDA-MB-231 cells. To confirm whether MZF1, ELK1, or their interaction contributes to the regulation of EMT, expression levels of EMT markers were examined in the Hs578T and MDA-MB-231 TNBC cell lines after knockdown of endogenous MZF1 or ELK1 by using shRNA. ELK1 knockdown had no alteration in the levels of the mesenchymal cell markers (Slug and Vimentin), or the epithelial cell marker, E-cadherin (Figure 3A). This result suggested that ELK1 was not a major contributor, that knockdown was not sufficient, and/or that redundant or overlapping signaling mechanisms contribute to EMT in Hs578T and MDA-MB-231 cells. On the other hand, although only a partial knockdown of MZF1 at the protein level was achieved, it was sufficient to impair EMT. Specifically, the mesenchymal markers Vimentin or Slug were down-regulated $(59.3 \%, p<0.01$ and $78.4 \%, p<0.01$, respectively) upon MZF1 knockdown (Figure 3F). Moreover, we then checked whether MZF1 knockdown is necessary for cell migration and invasion. Our data showed that MZF1 knockdown suppressed the migration capacity of Hs578T and MDA-MB-231cells, by $72.3 \%(p<0.01)$ and $70.7 \%(p<0.01)$, respectively. In addition, MZF1 knockdown reduced the invasion capacity of Hs578T and MDA-MB-231cells, by $79.4 \%(p<0.01)$ and $78.5 \%(p<0.01)$, respectively, but did not afect cell proliferation within 16 and 24 h (Figure 3G, H, I, J). Conversely, similar knockdown efficiency of ELK1 did not change their migration and invasion compared to the parental or vector control cells (Figure 3B,C,D,E). Besides, relatively fewer protein level of ELK1 had no influence on the MZF1 protein levels (Figure 3A). Thus, we speculated that the remaining amount of ELK1 protein is enough to sustain the forming of protein complex containing MZF1 and ELK1, and therefore the minimal stability threshold of MZF1 protein could be maintained.

To examine whether expression of exogenous MZF1 could rescue the epithelial phenotype in the MZF1-knockdown Hs578T and MDA-MB-231 cells, we applied exogenous MZF1 in these stable MZF1-knockdown cells (Figure 3F). Exogenous MZF1 counteracted the effects of MZF1-knockdown, since it increased the level of Slug (Hs578T-shMZF1: 2.89-fold, $p<0.05$; MDA-MB-231-shMZF1: 2.07-fold, $p>0.05$ ), compared to vehicle control (Figure 3F). Exogenous MZF1 also diminished the level of E-cadherin, by $85.5 \%(p<0.05)$ and $67.1 \%(p<0.05)$, respectively, in these MZF1-knockdown cells (Figure $3 \mathrm{~F}$ ). These findings are consistent with the results in Hs578T and MDA-MB-231 cells overexpressing MZF160-72. Taken together, these observations indicated that MZF1 modulated EMT in Hs578T and MDA-MB-231 cells.

MZF1 suppressed IGFIR expression by repressing its transcriptional level. Based on the above results, MZF1 has been shown to have a function in EMT, which is important in the progression of carcinoma. Depletion of the signaling molecule resulted in reversing EMT. Screening results from

Figure 3. MZF1 but not ELK1 is necessary for EMT in Hs578T and MDA-MB-231cells. Hs578T and MDA-MB-231 cells stably transfected with ELK1 shRNA (shELK1) were lysed, and western blot analysis of EMT markers was performed. " $(N)$ " indicates the nuclear fraction extracts, while the others are from whole cell lysates (A). The effect of ELK1-knockdown on cell proliferation after 16 and $24 \mathrm{~h}$ was determined via the MTT assay (Hs578T-V and MDA-MB-231-V cell absorbance values were considered as 100\%) (B) and immunoblotting with Ki-67 antibody $(C)$. Migration $(D)$ and invasion $(E)$ assays were performed on Hs578T and MDA-MB-231 cells stably expressing shELK1. In the same context, Hs578T and MDA-MB-231 cells stably expressing MZF1 shRNA (shMZF1) were lysed, and immunoblot analysis of EMT markers was performed $(F)$. MTT assay $(G)$ and immunoblotting for Ki-67 $(H)$ were used to determine the effect of MZF1-knockdown on cell proliferation. Migration (I) and invasion (J) assays were performed on Hs578T and $M D A-M B-231$ cells stably expressing shMZF1. The data represent the means $\pm S D$ from 3 separate experiments conducted in triplicate. $* * p<0.01$, compared to the control group. 


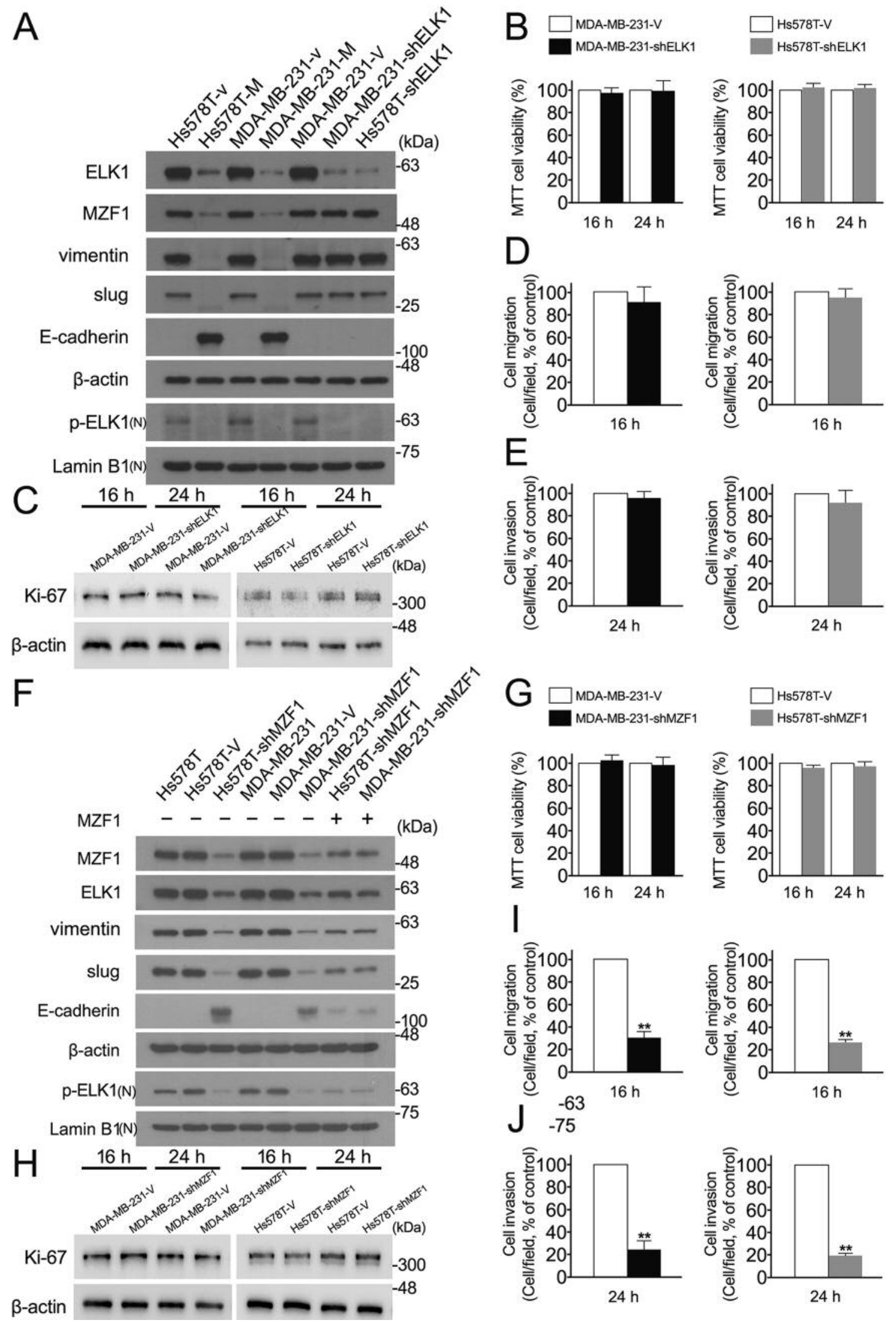


microarray assays have shown substantially higher level of the type 1 insulin-like growth factor receptor (IGF1R) tyrosine kinase in $\mathrm{MZF1}_{60-72}$-overexpressingHs578T and MDA-MB231cells (11). To check the hypothesis that IGF1R expression could be down-regulated by MZF1, the IGF1R expression was evaluated by immunoblot analysis in Hs578T and MDA-MB231cells stably overexpressing MZF1 $60-72$ and shMZF1. IGF1R expression was increased by 2.03 -fold $(p<0.01)$ and 3.08 -fold $(p<0.01)$ in Hs578T-MZF160-72 and MDA-MB-231-MZF16072 cells, respectively; while, by 4.13 -fold $(p<0.01)$ and 1.96fold $(p<0.01)$ in Hs578T-shMZF1 and MDA-MB-231-shMZF1 cells, respectively (Figure 4A). Moreover, overexpression of MZF1 decreased the IGF1R protein levels by $59.7 \%(p<0.01)$ and $48.4 \%(p<0.05)$, and RNA levels by $69.3 \%(p<0.01)$ and $55.6 \%(p<0.01)$, in MZF1-knockdown Hs578T and MDA-MB231cells, respectively (Figure $4 \mathrm{~A}$ and $\mathrm{B}$ ). These findings suggested that MZF1 reduced the expression of IGF1R in Hs578T and MDA-MB-231 cells.

The regulation of IGF1R transcription activity by MZF1 in cells stably expressing MZF160-72, shELK1 or shMZF1 was investigated. The promoter sequence of IGF1R gene was analyzed using the TRANSFAC database to identify the putative MZF1 binding sequence. Four potential binding sites for MZF1 located at nucleotides -299/-291, -138/-130, $+501 /+514$, and $+542 /+556$ were identified with the IGF1R promoter (Figure 4C). The IGF1R promoter fragment $(-765 /+583)$ was constructed with a luciferase reporter and was introduced into the Hs578T and MDA-MB-231 cells stably transfected to express MZF1 $60-72$, shELK1, shMZF1, or control vector. According to the results from the luciferase report assay, cells overexpressing $\mathrm{MZF}_{60-72}$ and MZF1-knockdown showed a minor increase in IGF1R promoter activity compared to the vector control and ELK1-knockdown cells (Figure 4C). These results indicated that MZF1 suppresses IGF1R promoter activity, but the same cannot be said for ELK1.

To confirm whether MZF1 binds directly to the promoter region of IGF1R, ChIP experiments were performed using MZF1 and ELK1 antibodies followed by screening whether the region of human IGF1R promoter is bound together. Immunoprecipitated chromatin DNA fragments from cells with vector or overexpressing $\mathrm{MZF1}_{60-72}$ were analyzed with PCR to identify the MZF1 binding regions in the IGF1R promoter. In Hs578T-MZF1 $1_{60-72}$ cells, the amount of PCR amplification specific for the MZF1 binding regions -299 to $-291,-138$ to -130 , and +501 to +556 , within the IGF1R promoter were decreased by $67.1 \%, 73.4 \%$, and $78.7 \%$, respectively, of PCR product compared to vector control cells (Figure 4D). The results from the re-ChIP assay indicated that ELK1 did not exist within this protein complex when MZF1 was bound to the IGF1R promoter. These findings indicated that MZF1 directly bound to the promoter region of IGF1R and repressed IGF1R transcription activity. However, the same cannot be said for ELK1.
IGF1R signaling through ER $\alpha$ regulated slug, E-cadherin and $E M T$. Recent evidence showed that two of the most important pathways in human breast cancer involve ER $\alpha$ signaling and Slug/E-cadherin, and the latter results in EMT (28). To investigate the effect of IGF1R on the expression of ER $\alpha$, MZF1 was knocked down in Hs578T and MDA-MB-231 cells and it was monitored whether endogenous $\mathrm{ER} \alpha$ is regulated by $\mathrm{MZF1/IGF1R.} \mathrm{A} \mathrm{dramatic} \mathrm{increase} \mathrm{in} \mathrm{ER} \alpha$ protein level was observed when IGF1R was up-regulated in Hs578T-shMZF1 and MDA-MB-231-shMZF1cells (Figure 4A). The results of MZF160-72-overexpressing Hs578T and MDA-MB-231 cells were similar (Figure 4E). In the stable knockdown cells, Hs578T-shMZF1 and MDA-MB-231-shMZF1, the slug protein was decreased. By contrast, the expression of E-cadherin was significantly increased (Figure 4A). In addition, to further examine the impact of IGF1R expression alterations on the protein level of ER $\alpha$, we performed transient transfection with the plasmid encoding full-length MZF1 in stable knockdown cells, Hs578T-shMZF1 and MDA-MB-231-shMZF1. Our rescue study showed that overexpression of the exogenous MZF1 in MZF1-knockdown cells resulted in the downregulation of IGF1R and ER $\alpha$ in Hs578T-shMZF1 (IGF1R: $61.8 \%, p<0.05$; $\mathrm{ER} \alpha: 49.6 \%, p<0.05)$ and MDA-MB-231-

Figure 4. MZF1 suppresses IGF1R expression and then induces EMT by directly binding to the promoter region of IGF1R. ( $A$ and $B)$ Transcriptional down-regulation of IGF1R gene expression by $M Z F 1$. Hs578T and MDA-MB-231 cells with stably expressing MZF1 shRNA constructs (Hs578T-shMZF1 and MDA-MB-231-shMZF1 cells) were transiently transfected with the plasmid expressing full-length MZF1 (shown as "MZF1 +"). The changes in the protein (A) and RNA levels (B) of MZF1-knockdown and rescue with exogenous MZF1 were measured via western blot and RT-PCR, respectively, compared to those of the parental cells. Four MZF1 binding elements were predicted on the IGF1R promoter region. The promoter plasmid construct, IGF1R-Luc, was transiently transfected into the Hs578T or MDA-MB-231 cells stably expressing $M Z F 1_{60-72}$, shELK1, shMZF1 or control vector to measure the reporter activity via luciferase assay $(C)$. The binding ability of $M Z F 1$ to the IGF1R promoter was evaluated via ChIP and re-ChIP analysis in MZF1 60-72-expressing Hs578T cells (Hs578T-M) cells. ChIP using antiMZF1 antibody was performed on chromatin extracted from cells expressing $\mathrm{MZF1}_{60-72}$ (Hs578T-M) or control vector (Hs578T-v), and three specific IGF1R promoter regions (ChIP primers $a, b$, and $c$ ) were amplified by $P C R$, respectively. Input samples were used as positive control (D). The effects of the IGF1R inhibitor, AG-1024, on the expression of IGF1R and its downstream signaling pathway were examined in $M D A-M B-231$ and $H_{s} 578 T$ cells stably expressing $M Z F 1_{60}$ 72 or control vector, as well as in MCF-7 cells. The cells were treated with $1 \mu M A G-1024$ for $3 d$, and the levels of IGF1R, ER $\alpha$, slug, and Ecadherin were assessed via western blot. " $(N)$ " indicates the nuclear fraction extracts, otherwise whole cell lysates are analyzed (E). The effects of the p38 MAPK inhibitor, SB203580, on the expression of IGF1R and its downstream signaling pathway were examined in MDA-MB-231 and $H_{5} 578 T$ cells stably expressing $M_{Z F 1_{60-72}}$ or control vector, as well as in MCF-7 cells. The cells were cultured in the presence of SB203580 for $8 \mathrm{~h}$ and were then subsequently analyzed via immunoblot analysis $(F)$. 


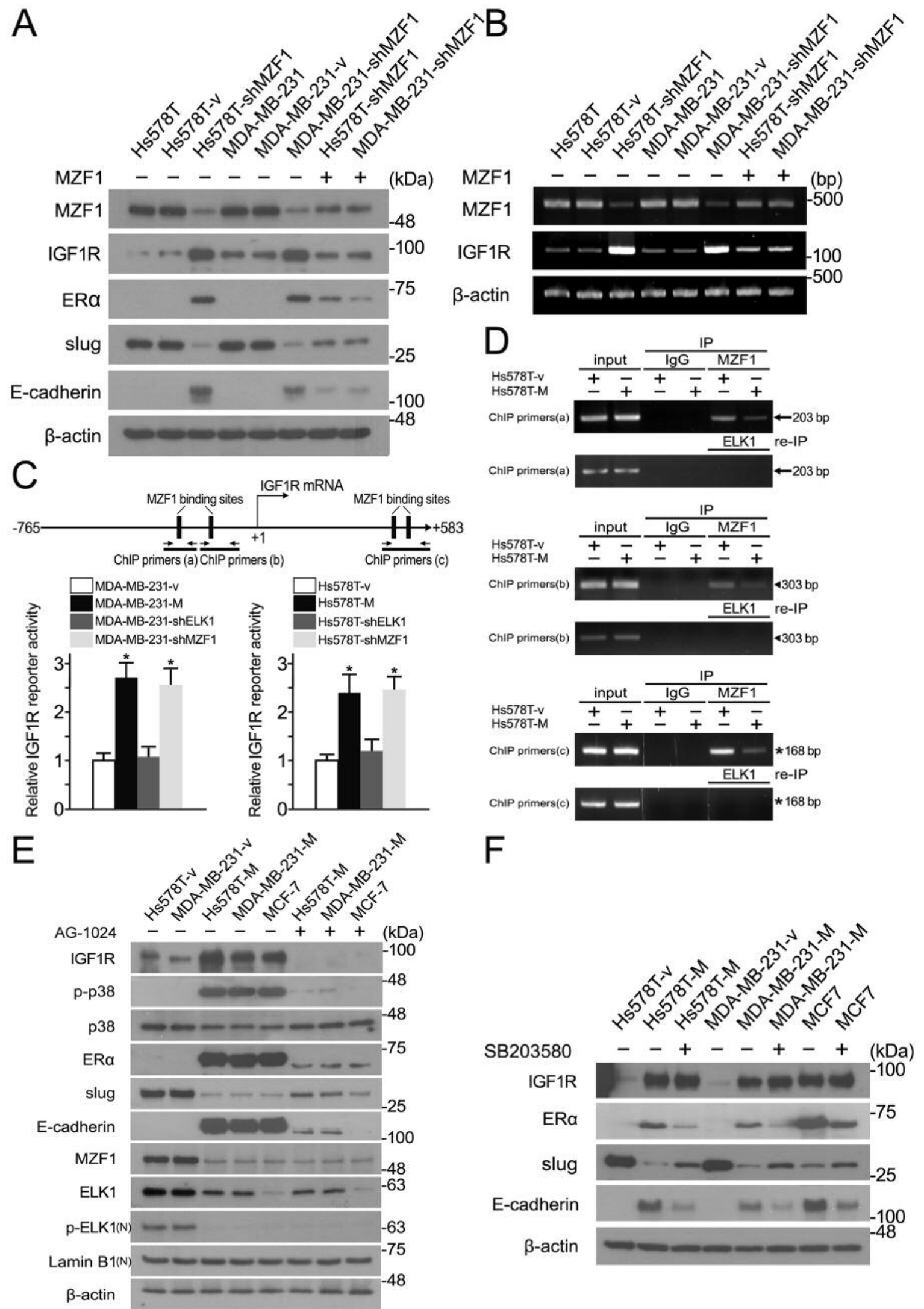


shMZF1 cells (IGF1R: $50.6 \%, p<0.05 \mathrm{ER} \alpha: 75.6 \%, p<0.01$ ) (Figure 4A). These findings also indicated that a significant correlation existed between IGF1R and ER $\alpha$ in Hs578TMZF1 $_{60-72}(\mathrm{r}=0.583, p<0.001)$ and MDA-MB-231-MZF1 ${ }_{60-72}$ $(\mathrm{r}=0.489, p<0.001)$ cells by analyzing with the Pearson's correlation coefficient. The relationship between IGF1R and $\mathrm{ER} \alpha$ appeared to serve an important function in the regulation of EMT; thus, we examined the effect of the IGF1R inhibitor, AG-1024, on the link of these proteins. AG-1024 impaired the activation of IGF1R and ER $\alpha$ in cells overexpressing $\mathrm{MZF}_{60}$ 72 (Figure 4E). Similar results were obtained with the treatment of the IGF1R inhibitor from MCF7 or MZF1-knockdown of Hs578T cells (Figure 4E). Furthermore, the inhibition of IGF1R by treatment with AG-1024 also restored slug expression. These findings indicated that the inhibition of IGF1R could tend to be mesenchymal phenotype in $\mathrm{MZF}_{60}$ 72-overexpressing Hs578T and MDA-MB-231 cells.

p38 MAPK was prominently activated by IGFIR. Downstream networks of activated IGF1R included the Ras/Raf/MEK/ERK and PI3K/Akt pathways. Moreover, two other mitogen-activated protein (MAP) kinases, namely, p38 and JNK, were activated (29). To investigate the molecular mechanism by which IGF1R selectively influenced migration and invasion, we examined the phosphorylation levels of MAPK family members in MZF1 ${ }_{60-}$ 72-overexpressing Hs578T cells (Figure 4E). The phosphorylation level of p38 was significantly increased in MZF1 ${ }_{60-72}$-overexpressing Hs578T and MDA-MB-231 cells (9.26-fold, $p<0.01$ and 5.47-fold, $p<0.01$, respectively; data not shown). This change suggested that the activation of $\mathrm{p} 38$ MAPK maybe involved in the down-regulation of MZF1induced transition to epithelial potential in TNBC cells. To investigate the hypothesis, we subjected Hs578T and MDAMB-231 cells to treatment with SB203580, the p38 MAPK inhibitor. SB203580 markedly down-regulated the expressions of ER $\alpha$ and E-cadherin by $68 \%(p<0.05)$ and $60.5 \%(p<0.01)$, and by $59.7 \%(p<0.05)$ and $64.6 \%(p<0.05)$ in Hs578T$\mathrm{MZF}_{60-72}$ and MDA-MB-231-MZF1 $1_{60-72}$ cells, respectively (Figure 4F). Treatment with SB203580 up-regulated the expression of slug in $\mathrm{MZF}_{60-72}$-overexpressing Hs578T and MDA-MB-231 cells. The protein levels of MZF1, ELK1, and IGF1R had no distinct changes compared to SB203580 treatment, indicating that these proteins might be upstream components of the signaling pathway. Moreover, the inhibition of IGF1R with AG-1024 reduced the phosphorylation level of p38, and the protein expression levels of ER $\alpha$ and E-cadherin in MZF1-knockdown Hs578T (reduced by $88.9 \%, p<0.001$; $84 \%, p<0.001 ; 83.2 \%, p<0.001$, respectively) and MDA-MB231 cells (reduced by $81.1 \%, p<0.001 ; 80.3 \%, p<0.001 ; 81.3 \%$, $p<0.001$, respectively), as well as in MCF7 cells (reduced by $90.7 \%, p<0.01 ; 82.6 \%, p<0.01 ; 98.1 \%, p<0.05$, respectively) (Figure 4E). Taken together, these results suggested that p38 MAPK would be a critical and necessary downstream target of

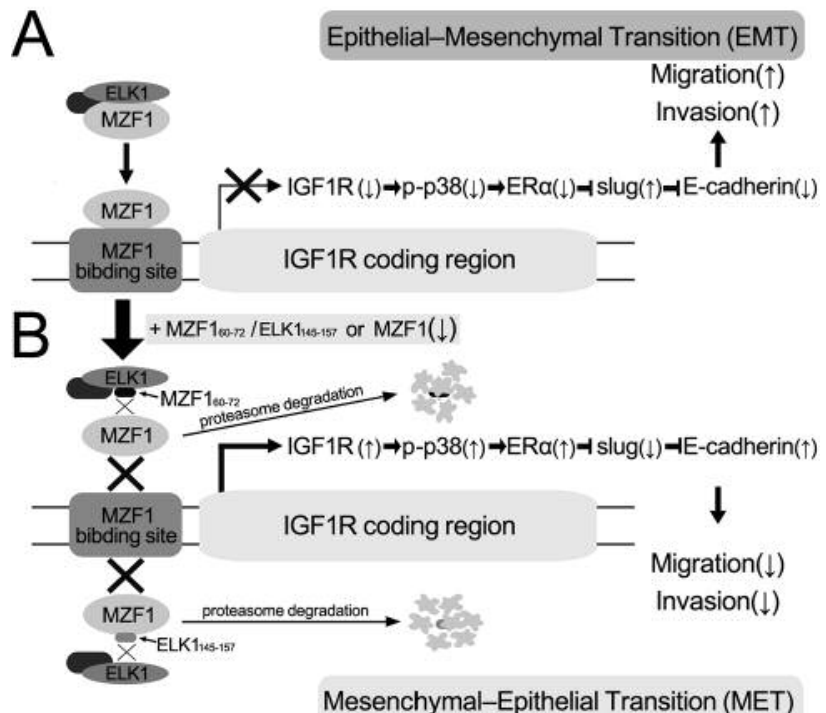

Figure 5. Disrupting the interaction between MZF1 and IGF1R promoter increases the epithelial potential. A scheme depicts the regulation of IGF1R expression by interaction with $M Z F 1$. The results showed that MZF1 but not ELK1 interacts with the IGF1R promoter region to regulate $I G F 1 R$ expression $(A)$. Interrupting their interactions by the $M Z F 1_{60-72}$ peptide led to decreased protein-DNA binding activity, followed by up-regulating IGF1R expression, and eventually attenuated EMT potential and tumorigenesis $(B)$.

IGF1R for the regulation of EMT. Treatment with p38 MAPK inhibitor would effectively prevent the down-regulation of MZF1-induced transition to epithelial potential in Hs578T and MDA-MB-231 cells.

Degradation of MZF1 attenuates malignant phenotypes of TNBC cells. We examined the effects of MZF $1_{60-72}$ on the tumorigenic potential of Hs578T and MDA-MB-231 cells. Cell migration and invasion were significantly reduced by $87.3 \%(p<0.01)$ and $89.1 \%(p<0.01)$, respectively, in $\mathrm{MZF}_{60-72}$-expressing stable cells relative to parental and control cells (Figure 2B). However, no changes in cell proliferation were observed. We performed ChIP and luciferase reporter assay to identify MZF1-responsive elements in the IGF1R promoter, thereby confirming the signaling pathway involved. Compared with its non-genomic fashion through ligand/receptor bonding, our evidence indicated that the IGF1R activity is regulated by MZF1 via IGF1R genomic (transcriptional) mechanism. The present work revealed that MZF1 was involved in slug/E-cadherin signaling through IGF1R/p38 MAPK/ER $\alpha$ signaling pathway, with the latter leading to EMT in malignant cells. Therefore, disruption of MZF1 and ELK1 complex formation attenuated MZF1-mediated cancer progression. The inhibitory effects of $M Z F 1_{60-72}$ on tumorigenesis further demonstrated that the 
fragment of MZF1 was essential for the formation of the MZF1/ELK1 interaction and transcriptional repression of IGF1R gene promoter, which is scarcely expressed in TNBC cells (Figure 5). These data indicate that MZF1 directly binds to the IGF1R promoter to suppress its transcription, thus inhibiting the invasion and metastasis of Hs578T and MDAMB-231 cells, suggesting the tumor suppressive functions of MZF1 in the progression of TNBC.

\section{Discussion}

In this study, we validated that MZF1 bound with ELK1 in high-level MZF1-expressing TNBC cells. We also demonstrated that MZF1 loses its stability to be a labile protein with a shorter half-life and its degradation is mediated through the proteasome pathway in $\mathrm{MZF}_{60-72}$-overexpressing Hs578T and MDA-MB-231 cells. We then examined whether $\mathrm{MZF1}_{60-72}$ overexpression can affect EMT in TNBC cells. Based on the results of cycloheximide decay assays, we hypothesized that $\mathrm{MZF}_{60-72}$ functioned as a competitor and interacted with endogenous protein complex containing MZF1/ELK1. The MZF1 $60-72$-mediated effects on cell phenotype were characterized by morphological changes, decreased expression of mesenchymal markers, and increased expression of the epithelial marker, E-cadherin. $\mathrm{MZF}_{60-72}$ interacted with the heparin-binding domain of ELK1, and subsequently this interaction caused instability and degradation of endogenous MZF1 protein in a proteasomedependent manner by competitively disrupting the interaction between MZF1 and ELK1 (Figure 2E). Moreover, MZF1 lost its stability and was degraded in a proteasome-dependent manner. Our observations suggested that $M Z F 1_{60-72}$ competitively took over the binding domains between endogenous MZF1 and ELK1. This is because MZF1 $60-72$ destroyed the original folding of MZF1 assisted by ELK1, thereby resulting in the eventual degradation of MZF1.

ELK1 transcription factor, is a downstream transcriptional target of MAPK, and has been considered to be localized predominantly in the nucleus. Once phosphorylated on Ser383/Ser389, ELK-1 undergoes conformational changes leading to increased DNA-binding and transcriptional activity $(30,31)$. Herein, ELK1 was located in both nuclear and cytoplasmic compartments in breast cancer cells, and the data obtained in this study demonstrated that inhibition of the ELK1-MZF1 interaction reduced the phosphorylation level of ELK1 in the nucleus of $\mathrm{MZF}_{60-72}$-overexpressing Hs578T and MDA-MB-231 cells (Figure 2C). Returning the expression of MZF1 to its original protein level reversed ELK1 nuclear phosphorylation, thereby indicating that ELK1 nuclear phosphorylation was directly linked to the protein amount of MZF1 (Figure 3F). This result also suggested that the interaction of ELK1 and MZF1 may be involved in the nuclear translocation of ELK1 in TNBC cells.
Based on our results on the $\mathrm{MZF1}_{60-72}$-mediated transition to epithelial-like state, MZF1 was a critical regulator of EMT in TNBC cells, but not ELK1 (Figure 3). In addition, we showed that a previously unidentified mechanism in transcriptional regulation contributed to the EMT in TNBC cells expressing MZF1. Our results from the Western blot analysis and RT-PCR experiments showed a distinct downregulation of IGF1R mRNA and protein levels in cells overexpressing MZF1, thereby confirming that the transcription factor MZF1 functioned as a transcription repressor of IGF1R expression in Hs578T and MDA-MB231 cells (Figure 4A and B). The ectopic expression of MZF1 was associated with increased the mesenchymal marker expression and decreased the expression of the epithelial marker (Figure 4A). Subsequently, we demonstrated that MZF1, but not ELK1, inhibited the expression of IGF1R by directly binding to the IGF1R promoter region and suppressing the IGF1R transcription activity (Figure 4C, D). Similar results have also been found in studies of MZF1 being capable of down-regulating IGFIR gene expression by binding directly to its promoter and 5 'regionin NPM-ALK ${ }^{+}$T-cell lymphoma (9).

MZF1, a 485-amino acid protein belonging to the $\mathrm{C} 2 \mathrm{H} 2$ zinc finger gene family, contains 13 zinc fingers domains divided into two groups, as follows: one location contains zinc finger 1-4 domains, and the other in the carboxyl terminus contains zinc fingers 5-13 domains, which can bind DNA independently. Both zinc fingers have the ability to bind DNA, and DNA consensus binding sites have been identified for each of these DNA-binding domains (32). We assumed that MZF1 has the potential to bind with sequences located both upstream and downstream of the transcription start site between -299 and +556 of the IGFIR gene. We then performed luciferase activity assay and ChIP analysis to confirm that MZF1, but not ELK1, could bind to the sequences within the IGF1R promoter region. As a possible explanation for the results from our experiments, MZF1 did not combine with ELK1 while MZF1 bound to the promoter region of IGF1R gene.

Recent evidence shows that transcriptional factor MZF1 activates respective hub genes mediating the conscription and commencement of cancer by phosphorylation and sumoylation which are considered as post-translational modification (33). As with the heterologous DNA binding domain, MZF1 determines its transcriptional regulatory function may depend on the presence of tissue-specific regulators/adapters or differential MZF1 modifications. Therefore, MZF1 has been considered as a bi-functional transcription regulator that can act as both a transcription activator and a transcription repressor via influencing insulin or via regulating the MAPK cascade, RPS6K and PTEN signaling $(32,34)$. Overexpression of MZF1 leads to the transactivation of Axl promoter and induces the migration and invasion of solid tumor cells (34). In contrast, MZF1 
could be a tumor suppressor in tumorigenesis. MZF1 reduces the migratory capability and invasiveness of cervical cancer cells through suppressing matrixmetalloproteinase-2 expression (34). Moreover, Ikaros isoform 1 (Ik1) and MZF1 directly bind to the promoter of $I G F 1 R$ to suppress its transcription and the pathogenesis of nucleophosminanaplastic lymphoma kinase oncogenic protein (NPM-ALK)positive T-cell lymphoma (9). In this study, we demonstrated for the first time that MZF1 inhibited IGF1R promoter activity and gene expression, thereby resulting in the induced migration and invasion of TNBC as oncogenic inducer. We also illustrated that IGF1R was a novel downstream gene of MZF1 in TNBC. First, the expression of MZF1 and IGF1R was positively correlated in Hs578T and MDA-MB-231 cells. Second, endogenous IGF1R expression, both protein and transcription, was decreased or increased by overexpression or knockdown of MZF1 in Hs578T and MDA-MB-231 cells. Third, the luciferase activity of IGF1R promoter was responsive to MZF1 knockdown. Finally, ChIP assay revealed the binding of MZF1 to IGF1R promoter, recommending that MZF1 could restrain the IGF1R expression by suppressing transcription.

Previous reports have demonstrated that both IGF1R and its downstream adaptor insulin receptor substrate 1 (IRS1) are estrogen-regulated genes $(35,36)$. Furthermore, IGF1R can activate estrogen receptor (37). However, the functions and downstream gene of IGF1R in Hs578T and MDA-MB231 cells still remain to be elucidated. In this study, we observed that IGF1R signal activation resulted in the upregulation of $E R \alpha$ gene expression by activating the p38 MAPK signaling pathway (data not shown). In line with these observations, the inhibition of IGF1R appeared to decrease the phosphorylation level of p38 in the stably expressing MZF1 $60-72$ Hs578T, MDA-MB-231 or MCF7 cells (Figure 4E). Moreover, the expression of ER $\alpha$ was down-regulated after treatment with the inhibitor of IGF1R. Similarly, the protein level of IGF1R in $\mathrm{MZF}_{60-72^{-}}$ overexpressing cells still maintained in the presence of the inhibitor of p38 MAPK (SB203580) which significantly decreased the levels of ER $\alpha$ and E-cadherin. In contrast to these two proteins, the level of slug increased in these two TNBC cells under the same treatment condition (Figure 4F). Thus, we suggested that the p38 MAPK pathway would be necessary for IGF1R in modulating MET. These results were consistent with previous studies demonstrating that IGF signaling pathway and ER $\alpha$ performing dynamic and intricate crosstalk, resulting in bidirectional regulation of expression and activity $(6,38)$. Furthermore, ER $\alpha$ could directly affect slug transcription and then regulate E-cadherin and EMT (27). Herein, our findings showed that the increase in $\mathrm{ER} \alpha$ expression was associated with a distinct decrease in slug after $M Z F 1_{60-72}$ transfection, and the abrogation of ER $\alpha$ expression rescued the expression of slug in Hs578T and
MDA-MB-231 cells with stably expressing MZF1 $60-72$ (Figure 4E and F).

Given that transcription factors are increasingly redescribed as 'undrugged', instead of its previous description as 'undruggable', research on transcription factor-inhibiting peptides, such as those that inhibit Notch1 activity and rescue p53 activity, and modification of protein-protein interactions would pave the way for new avenues of drug research and cancer treatment (39-42). In the present study, we used regulatory protein-fused peptides containing transcription factor MZF1 or ELK1 truncation as a novel inhibitor to target the protein-protein interface by saturating their binding site and triggering endogenous MZF1 degradation. In conclusion, our results provided strong evidence that the degradation of MZF1 contributed to reinstate of IGF1R expression in TNBC cells, thereby inducing the downstream expression of signaling molecules, including p38 MAPK and ER $\alpha$, and then ER $\alpha$ through slug regulating E-cadherin and EMT. However, we could not completely exclude the possibility that the outcome of degradation of MZF1 in TNBC was not only mediated through the up-regulation of IGFIR because this transcription factor may be involved in the regulation of other survival/oncogenic signaling mechanisms. This study revealed that invasion- and EMT- associated genes were upregulated in TNBC with a high expression of MZF1. These observations suggested that MZF1 might function as a potential tumor inducer and contribute to the progression of TNBC metastasis. Therefore, considering the high correlation between MZF1 and ELK1 transcription factors in TNBC patients, inhibiting the ELK1/MZF1 interaction represented a novel and feasible strategy to specifically impair EMT through transactivating IGF1R downstream signaling, thereby suggesting its potency for development as an alternative anti-cancer strategy.

\section{Conclusion}

In Hs578T and MDA-MB-231 cells, MZF1 is highly expressed and it directly binds to the IGF1R promoter to suppress its transcription. IGF1R could alter slug-mediated EMT and is influenced by MZF1. MZF1 is thought to be a negative regulator of IGF1R-p38MAPK-ER $\alpha$-slug-E-cadherin signaling in high-level MZF1-expressing TNBC cells, and associated with tumor invasion and metastasis. Herein, we investigated whether MZF1 strengthens the mesenchymal phenotype by de-repressing slug expression and function through inhibition of IGF1R and ER $\alpha$ expression. We have shown that expression of slug is reduced during endogenous MZF1 degradation in Hs578T and MDA-MB-231 cells. Knockdown of MZF1 or overexpression of the acidic domain of MZF1 shifts initial mesenchymal phenotype that alters cellular morphology from spindle-like into cobblestone-like 
shape. Treatment with IGF1R tyrosine kinase inhibitor, AG1024 , or the p38 MAPK inhibitor, SB203580 partially restores slug expression levels in these two cell lines. In addition, expression of MZF1 would rescue the phenotype. Thus, MZF1 modulates the motility and invasion potential of high-level MZF1-expressing TNBC cells and acts through the suppression of IGF1R by directly binding to the IGF1R promoter (Figure 5). Therefore, it might be therapeutically beneficial to promote the epithelial phenotype in TNBC. To the best of our knowledge, this is the first report to indicate the role of MZF1 in these cascades, suggesting this protein as a potential target for the development of new therapeutic approaches for the TNBC patients expressing MZF1.

\section{Conflicts of Interest}

All Authors declare that they have no conflicts of interest.

\section{Authors' Contributions}

Conception JYL and CJL; Funding acquisition, CHY, KIL, KTT and CSC; Investigation, CWH, KTT, FMH, YRP and CJL; Resources, JYL and CSC; Methodology and validation, CJL and YRP; Supervision, KIL and JYL; Writing - original draft, FMH., CJL and CHY; Writing - review and editing, CJL; All authors read and approved the final manuscript.

\section{Acknowledgements}

This work was supported by the Research Fund of Tung's Taichung MetroHarbor Hospital (TTMHH-107R0011, TTMHH-107R0012 and TTMHH-108R0026) and Chang Bing Show Chwan Memorial Hospital (RD105036).

\section{References}

1 Jemal A, Siegel R, Xu J and Ward E: Cancer statistics, 2010. CA Cancer J Clin 60: 277-300, 2010. PMID: 20610543. DOI: 10.3322/caac.20073

2 Yu JK, Yue CH, Pan YR, Chiu YW, Liu JY, Lin KI and Lee CJ: Isochlorogenic acid c reverses epithelial-mesenchymal transition via down-regulation of EGFR pathway in MDA-MB-231 cells Anticancer Res 38: 2127-2135, 2018. PMID: 29599331. DOI: 10.21873/anticanres. 12453

3 Dent R, Trudeau M, Pritchard KI, Hanna WM, Kahn HK, Sawka CA, Lickley LA, Rawlinson E, Sun P and Narod SA: Triplenegative breast cancer: clinical features and patterns of recurrence. Clin Cancer Res 13: 4429-4434, 2007. PMID: 17671126. DOI: 10.1158/1078-0432.CCR-06-3045

4 Carey L, Winer E, Viale G, Cameron D and Gianni L: Triplenegative breast cancer: disease entity or title of convenience? Nat Rev Clin Oncol 7: 683-692, 2010. PMID: 20877296. DOI: 10.1038/nrclinonc. 2010.154

5 Li H, Batth IS, Qu X, Xu L, Song N, Wang R and Liu Y: IGF$1 \mathrm{R}$ signaling in epithelial to mesenchymal transition and targeting IGF-IR therapy: overview and new insights. Mol Cancer 16: 6, 2017. PMID: 28137302. DOI: 10.1186/s12943-016-0576-5
6 Farabaugh SM, Boone DN and Lee AV: Role of IGF1R in breast cancer subtypes, stemness, and lineage differentiation. Front Endocrinol 6: 59, 2015. PMID: 25964777 DOI: 10.3389/fendo. 2015.00059

7 Katzenellenbogen BS and Norman MJ: Multihormonal regulation of the progesterone receptor in MCF-7 human breast cancer cells: interrelationships among insulin/insulin-like growth factor-I, serum, and estrogen. Endocrinol 126: 891-898, 1990. PMID: 2404751. DOI: 10.1210/endo-126-2-891

8 Sarfstein R, Maor S, Reizner N, Abramovitch S and Werner H: Transcriptional regulation of the insulin-like growth factor-I receptor gene in breast cancer. Mol Cell Endocrinol 252: 241246, 2006. PMID: 16647191. DOI: 10.1016/j.mce.2006.03.018

9 Vishwamitra D, Curry CV, Alkan S, Song YH, Gallick GE, Kaseb AO, Shi P and Amin HM: The transcription factors Ik-1 and MZF1 downregulate IGF-IR expression in NPM-ALK (+) T-cell lymphoma. Mol Cancer 14: 53, 2015. PMID: 25884514. DOI: 10.1186/s12943-015-0324-2

10 Weber CE, Kothari AN, Wai PY, Li NY, Driver J, Zapf MA, Franzen CA, Gupta GN, Osipo C and Zlobin A: Osteopontin mediates an MZF1-TGF- $\beta 1$-dependent transformation of mesenchymal stem cells into cancer-associated fibroblasts in breast cancer. Oncogene 34: 4821-4833, 2015. PMID: 25531323. DOI: $10.1038 /$ onc. 2014.410

11 Lee CJ, Hsu LS, Yue CH, Lin H, Chiu YW, Lin YY, Huang CY, Hung MC and Liu JY: MZF-1/ELK-1 interaction domain as therapeutic target for protein kinase $\mathrm{C} \alpha$-based triple-negative breast cancer cells. Oncotarget 7: 59845-59859, 2016. PMID: 27542222. DOI: $10.18632 /$ oncotarget.11337

12 Ko H, Kim S, Yang K and Kim K: Phosphorylation-dependent stabilization of MZF1 upregulates N-cadherin expression during protein kinase CK2-mediated epithelial-mesenchymal transition. Oncogenesis 7: 27, 2018. PMID: 29540671. DOI: 10.1038/ s41389-018-0035-9

13 Liu F, Gu L, Shan B, Geng CZ and Sang MX: Biomarkers for EMT and MET in breast cancer: An update. Oncology Letters 12: 4869-4876, 2016. PMID: 28105194. DOI: 10.3892/ ol.2016. 5369

14 Gyorffy B, Lanczky A, Eklund AC, Denkert C, Budczies J, Li Q and Szallasi Z: An online survival analysis tool to rapidly assess the effect of 22,277 genes on breast cancer prognosis using microarray data of 1809 patients. Breast Cancer Res Treatment 123: 725-731, 2010. PMID: 20020197. DOI: 10.1007/s10549009-0674-9

15 Chang IC, Chiang TI, Lo C, Lai YH, Yue CH, Liu JY, Hsu LS and Lee CJ: Anemone altaica induces apoptosis in human osteosarcoma cells. American J Chinese Med 43: 1031-1042, 2015. PMID: 26224029. DOI: 10.1142/S0192415X15500597

16 Lee CJ, Yue CH, Lin YY, Wu JC and Liu JY: Antitumor activity of acriflavine in human hepatocellular carcinoma cells. Anticancer Res 34: 3549-3556, 2014. PMID: 24982368.

$17 \mathrm{Wu}$ TT, Hsieh YH, Hsieh YS and Liu JY: Reduction of PKC alpha decreases cell proliferation, migration, and invasion of human malignant hepatocellular carcinoma. J Cell Biochem 103: 9-20, 2008. PMID: 17486587. DOI: 10.1002/jcb.21378

18 Reid G, Hübner MR, Métivier R, Brand H, Denger S, Manu D, Beaudouin J, Ellenberg J and Gannon F: Cyclic, proteasomemediated turnover of unliganded and liganded ERalpha on responsive promoters is an integral feature of estrogen signaling. Mol Cell 11: 695-707, 2003. PMID: 12667452. 
19 Lin S, Wang X, Pan Y, Tian R, Lin B, Jiang G, Chen K, He Y, Zhang $\mathrm{L}$ and Zhai W: Transcription factor myeloid Zinc-finger 1 suppresses human gastric carcinogenesis by interacting with metallothionein 2A. Clin Cancer Res 25: 1050-1062, 2019. PMID: 30301827. DOI: 10.1158/1078-0432.CCR-18-1281

20 Nurwidya F, Takahashi F, Kobayashi I, Murakami A, Kato M, Minakata K, Nara T, Hashimoto M, Yagishita S and Baskoro H: Treatment with insulin-like growth factor 1 receptor inhibitor reverses hypoxia-induced epithelial-mesenchymal transition in nonsmall cell lung cancer. Biochem Biophys Res Commun 455: 332338, 2014. PMID: 25446090. DOI: 10.1016/j.bbrc.2014. 11.014

21 MacPherson MR, Molina P, Souchelnytskyi S, Wernstedt C, Martin-Pérez J, Portillo F and Cano A: Phosphorylation of serine 11 and serine 92 as new positive regulators of human Snail1 function: potential involvement of casein kinase-2 and the cAMPactivated kinase protein kinase A. Mol Biol Cell 21: 244-253, 2010. PMID: 19923321. DOI: 10.1091/mbc.e09-06-0504

22 Ye JC, Hsu LS, Tsai JH, Yang HL, Hsiao MW, Hwang JM, Lee CJ and Liu JY: MZF-1/ELK-1/PKC $\alpha$ is associated with poor prognosis in patients with hepatocellular carcinoma. J Cancer 8 : 3028-3036, 2017. PMID: 28928894. DOI: 10.7150/jca.20467

23 Peterson MJ and Morris JF: Human myeloid zinc finger gene MZF produces multiple transcripts and encodes a SCAN box protein. Gene 254: 105-118, 2000. PMID: 10974541.

24 Urruticoechea A, Smith IE and Dowsett M: Proliferation marker Ki-67 in early breast cancer. J Clin Oncol 23: 7212-7220, 2005. PMID: 10974541.

25 Hsu YL, Hou MF, Kuo PL, Huang YF and Tsai EM: Breast tumor associated osteoblast-derived CXCL5 increases cancer progression by ERK/MSK1/ELK-1/snail signaling pathway. Oncogene 32: 4436-4447, 2013. PMID: 23045282. DOI: 10.1038/onc.2012.444

26 Hajra KM, Chen DY and Fearon ER: The SLUG zinc-finger protein represses E-cadherin in breast cancer. Cancer Res 62: 1613-1618, 2002. PMID: 11912130.

27 Ye Y, Xiao Y, Wang W, Yearsley K, Gao JX, Shetuni B and Barsky SH: ER $\alpha$ signaling through slug regulates E-cadherin and EMT. Oncogene 29: 1451-1462, 2010. PMID: 20101232. DOI: 10.1038/onc. 2009.433

28 Hematulin A: Insulin-like growth factor 1 signalling and its role in cell survival and radiation sensitivity. Siriraj Med J 62: $263-$ 267, 2010 .

29 Hospital VI, Nishi E, Klagsbrun M, Cohen P, Seidah NG and Prat A: The metalloendopeptidase nardilysin $(\mathrm{NRDc})$ is potently inhibited by heparin-binding epidermal growth factor-like growth factor. Biochem J 367: 229-238, 2002. PMID: 12095415. DOI: 10.1042/BJ20020822

30 Yang SH, Shore P, Willingham N, Lakey JH and Sharrocks AD: The mechanism of phosphorylation-inducible activation of the ETS-domain transcription factor ELK-1. EMBO J 18: 5666-5674, 1999. PMID: 10523309 . DOI: $10.1093 / \mathrm{emboj} / 18.20 .5666$

31 Morris JF, Hromas R and Rauscher FJ: Characterization of the DNA-binding properties of the myeloid zinc finger protein MZF1: two independent DNA-binding domains recognize two DNA consensus sequences with a common G-rich core. Mol Cell Biol 14: 1786-1795, 1994. PMID: 8114711. DOI: 10.1128/ mcb.14.3.1786

32 Eguchi T, Prince T, Wegiel B and Calderwood SK: Role and regulation of myeloid zinc finger protein 1 in cancer. J Cell Biochem 116: 2146-2154, 2015. PMID: 25903835. DOI: 10.1002/jcb.25203
33 Tsai SJ, Hwang JM, Hsieh SC, Ying TH and Hsieh YH: Overexpression of myeloid zinc finger 1 suppresses matrix metalloproteinase- 2 expression and reduces invasiveness of $\mathrm{SiHa}$ human cervical cancer cells. Biochem Biophys Res Commun 425: 462-467, 2012. PMID: 22846578. DOI: 10.1016/ j.bbrc. 2012.07.125

34 Mudduluru G, Vajkoczy P and Allgayer H: Myeloid zinc finger 1 induces migration, invasion, and in vivo metastasis through Axl gene expression in solid cancer. Mol Cancer Res 8: 159-169, 2010. PMID: 20145042. DOI: 10.1158/1541-7786.MCR-09-0326

35 Lee AV, Jackson JG, Gooch JL, Hilsenbeck SG, CoronadoHeinsohn E, Osborne CK and Yee D: Enhancement of insulinlike growth factor signaling in human breast cancer: estrogen regulation of insulin receptor substrate-1 expression in vitro and in vivo. Mol Endocrinol 13: 787-796, 1999. PMID: 10319328. DOI: 10.1210/mend.13.5.0274

36 Litzenburger BC, Creighton CJ, Tsimelzon A, Chan BT, Hilsenbeck SG, Wang T, Carboni JM, Gottardis MM, Huang F and Chang JC: High IGF-IR activity in triple-negative breast cancer cell lines and tumorgrafts correlates with sensitivity to anti-IGF-IR therapy. Clin Cancer Res 17: 2314-2327, 2011. PMID: 21177763. DOI: 10.1158/1078-0432.CCR-10-1903

37 Lee AV, Weng CN, Jackson JG and Yee D: Activation of estrogen receptor-mediated gene transcription by IGF-I in human breast cancer cells. J Endocrinol 152: 39-47, 1997. PMID: 9014838.

38 Rota LM, Albanito L, Shin ME, Goyeneche CL, Shushanov S, Gallagher EJ, LeRoith D, Lazzarino DA and Wood TL: IGF1R inhibition in mammary epithelia promotes canonical Wnt signaling and Wnt1-driven tumors. Cancer Res 74: 5668-5679, 2014. PMID: 25092896. DOI: 10.1158/0008-5472.CAN-14-0970

39 Brown CJ, Quah ST, Jong J, Goh AM, Chiam PC, Khoo KH, Choong ML, Lee MA, Yurlova L and Zolghadr K: Stapled peptides with improved potency and specificity that activate p53. ACS Chem Biol 8: 506-512, 2013. PMID: 23214419. DOI: $10.1021 / \mathrm{cb} 3005148$

40 Moellering RE, Cornejo M, Davis TN, Del Bianco C, Aster JC, Blacklow SC, Kung AL, Gilliland DG, Verdine GL and Bradner JE: Direct inhibition of the NOTCH transcription factor complex. Nature 462: 182-188, 2009. PMID: 19907488 DOI: 10.1038/nature08543

41 Yeh JE, Toniolo PA and Frank DA: Targeting transcription factors: promising new strategies for cancer therapy. Curr Opin Oncol 25: 652-658, 2013. PMID: 24048019. DOI: 10.1097/ 01.cco.0000432528.88101.1a

42 Cornmark L, Lønne GK, Jögi A and Larsson C: Protein kinase $\mathrm{C} \alpha$ suppresses the expression of STC1 in MDA-MB-231 breast cancer cells. Tumor Biol 32: 1023-1030, 2011. PMID: 21720730. DOI: $10.1007 / \mathrm{s} 13277-011-0205-2$
Received April 1, 2019

Revised June 29, 2019

Accepted July 4, 2019 